\title{
ON THE OPTIMIZATION OF BROADBAND PHOTOMETRY FOR GALAXY EVOLUTION STUDIES
}

\author{
A. GIL de PAZ ${ }^{1,2}$ AND B. F. MAdORE ${ }^{1,3}$ \\ Received 2001 June 19; accepted 2000 December 26
}

\begin{abstract}
We have derived the uncertainties to be expected in the derivation of galaxy physical properties (star formation history, age, metallicity, and reddening) when comparing broadband photometry to the predictions of evolutionary synthesis models. We have obtained synthetic colors for a large sample ( 9000) of artificial galaxies by assuming different star formation histories, ages, metallicities, reddening values, and redshifts. The colors derived have been perturbed by adopting different observing errors and compared against the evolutionary synthesis models grouped in different sets. The comparison has been performed using a combination of Monte Carlo simulations, a maximum likelihood estimator, and principal component analysis. After comparing the input and derived output values we have been able to compute the uncertainties and covariant degeneracies between the galaxy physical properties as a function of (1) the set of observables available, (2) the observing errors, and (3) the galaxy properties themselves. In this work we have considered different sets of observables, some of them including the standard Johnson-Cousins $\left(U B V R_{C} I_{C}\right)$ and Sloan Digital Sky Survey (SDSS) bands in the optical, the Two Micron All-Sky Survey (2MASS) bands in the nearinfrared, and the Galaxy Evolution Explorer (GALEX) bands in the UV, at three different redshifts, $z=0.0$, 0.7 , and 1.4. This study is intended to represent a basic tool for the design of future projects on galaxy evolution, allowing an estimate of the optimal bandpass combinations and signal-to-noise ratios required for a given scientific objective.
\end{abstract}

Key words: galaxies: evolution — galaxies: photometry — methods: numerical — methods: statistical

\section{INTRODUCTION}

The catalogs produced by wide-field and all-sky surveys currently under development (e.g., the Galaxy Evolution Explorer [GALEX], Martin et al. 1997; the Sloan Digital Sky Survey [SDSS], York et al. 2000; the Two Micron AllSky Survey [2MASS], Skrutskie et al. 1997; and DENIS [Deep Near-Infrared Survey of the Southern Sky], Epchtein et al. 1997), in combination with astronomical databases such as the NASA/IPAC Extragalactic Database, are beginning to provide easy access to extensive cross-correlated UV, optical, and near-infrared (NIR) photometry for millions of galaxies. The comparison of this huge amount of photometric data with the predictions of state-of-the-art galaxy population synthesis models provides an opportunity to obtain a more nearly complete picture of the evolution of galaxies. One obvious goal is to gain insight into the star formation history, as well as the chemical and dust content evolution of galaxies from the high- and intermediateredshift universe down to the present.

However, the reliability and precision of the derived galaxy properties expected to be found from these studies will depend on many factors; among these are (1) the number of bands and wavelength coverage of the available observations, (2) the measuring uncertainties, and (3) the degeneracies between the different galaxy properties given the available photometric bands. To discriminate between different scenarios of galaxy evolution, the comparison of photometric data and evolutionary synthesis models should

\footnotetext{
${ }^{1}$ NASA/IPAC Extragalactic Database, MS 100-22, California Institute of Technology, Pasadena, CA 91125; agpaz@ipac.caltech.edu, barry@ipac.caltech.edu.

2 Jet Propulsion Laboratory, MS 183-900, California Institute of Technology, Pasadena, CA 91109.

${ }^{3}$ The Observatories, Carnegie Institution of Astronomy, 813 Santa Barbara Street, Pasadena, CA 91101.
}

also include the quantification of the uncertainties and covariances between the galaxy properties derived. In addition, the use of different external inputs (evolutionary tracks, stellar atmospheres libraries, etc.) in the evolutionary synthesis models leads to various discrepancies in the output results that may also result in significant uncertainties in the properties derived. While a detailed study of these latter effects is beyond the scope of this paper, a nice approach to this problem may be found in Charlot, Worthey, \& Bressan (1996; see also Bruzual 2000; Cerviño, Luridiana, \& Castander 2000; Cerviño et al. 2001).

Some recent studies have incorporated the effects of the observing errors and flux calibration uncertainties in the determination of the properties of different galaxy samples (Gil de Paz et al. 2000, hereafter GIL00; Bell \& de Jong 2000; Brinchmann \& Ellis 2000). However, this type of analysis has not yet been performed in a systematic way, covering a large range of galaxy properties and/or star formation scenarios. A relevant exception is the work of Ronen, Aragón-Salamanca, \& Lahav (1999) on the principal component analysis of synthetic galaxy spectra. Similarly, no significant effort has yet been devoted to determining quantitatively the optimal set of observables and signal-to-noise ratio required to obtain reliably derived galaxy properties. Noteworthy exceptions are the works of Kodama, Bell, \& Bower (1999), Bolzonella, Miralles, \& Pelló (2000), and Wolf, Meisenheimer, \& Röser (2001) with regard to the galaxy classification and redshift determination in broadand medium-band surveys.

In this paper we explore the uncertainties expected in the derived properties of galaxies obtained from the analysis of broadband photometry of nearby, intermediate- and highredshift objects. We quantify these uncertainties by using a sample of artificially generated spectral energy distributions (converted to broadband colors) with known (input) physical properties, studying the dependence of the properties 
derived on the sets of bands available and the observing errors, as well as the galaxy star formation history and redshift. This work has interesting predictive capabilities and is intended to help in the design of future projects on the study of galaxy evolution. It can be used in the optimization of observing programs, helping to select the best wavelength bands and signal-to-noise ratios required to derive precise galaxy properties. This contribution is mainly focused on the study of the integrated properties of galaxies, star clusters, and $\mathrm{H}$ II regions, although it is our intent to generalize the application to spatially resolved portions of galaxies as well. A similar approach has been followed by Charlot \& Longhetti (2001) for the optimization of emission-line data in galaxy spectra, although no error analysis was carried out by those authors.

In $\S 2$ of this paper we briefly describe the synthetic galaxy sample. The procedure followed for determining the uncertainties and degeneracies between the physical properties in this sample is described in $\S 3$. Section 4 includes a detailed description of the results from this analysis. The main conclusions are given in $\S 5$. Finally, some future applications for this work are given in $\S 6$.

\section{SAMPLE}

We have generated a large number of synthetic galaxy colors parameterized by different star formation histories, metallicities, reddenings, and redshifts. Because of the unbounded number of possible combinations we have made some choices and simplifications.

With regard to their star formation history we have considered galaxies with exponential star formation having timescales $(\tau)$ between 0.2 and 6 Gyr. Although this scenario is a rough approximation to the actual star formation histories, it constitutes the most widely accepted parameterization of the star formation history in individual galaxies (see, e.g., Brinchmann \& Ellis 2000).

Thus, the properties to be determined are the timescale for the galaxy formation, the age of the galaxy, the stellar metallicity, and the reddening. A total of 9000 synthetic galaxies were generated, a third of them at redshift $z=0.0$, another third at $z=0.7$, and the remainder at redshift $z=1.4$. These redshift values were chosen to cover the epoch when most of the evolution of the star formation activity in the universe has apparently taken place (Gallego et al. 1995; Madau et al. 1996; Connolly et al. 1997; Madau, Dickinson, \& Pozzeti 1998). Since the models available provide only discrete values in metallicity we chose to assign to each galaxy the nearest metallicity value of those given by the corresponding model. The ranges of physical properties covered by the galaxies in the sample are shown in Table 1 .

Although a complete study of the effects of the model uncertainties on the galaxy properties derived is beyond the scope of this paper, the impact of the errors in the broadband colors due to stellar evolution prescription and spectral calibration uncertainties (Charlot et al. 1996; Yi, Demarque, \& Oemler 1997) will be taken into account in our further analysis. In addition, to illustrate the effect of these uncertainties we have considered two different sets of evolutionary synthesis models. The synthetic galaxies were generated using the predictions for the stellar continuum given by the GISSEL99 models (Bruzual \& Charlot 2002), while the best-fitting set of properties has been derived using both the GISSEL99 and the PÉGASE models (Version 2.0;
TABLE 1

Range of Galaxy Properties Considered

\begin{tabular}{lcc}
\hline \hline \multicolumn{1}{c}{ Parameter } & Sample $^{1}$ & Models $^{2}$ \\
\hline$E(B-V)_{\text {stl }}(\mathrm{mag}) \ldots \ldots \ldots$. & $0.00-1.00$ & $0.00-1.05$ \\
$\tau(\mathrm{Gyr}) \ldots \ldots \ldots \ldots \ldots \ldots$. & $0.2-6$ & $0.01-10$ \\
Age for $z=0 \ldots \ldots \ldots \ldots$. & $4-12 \mathrm{Gyr}$ & $1-15 \mathrm{Gyr}$ \\
Age for $z=0.7 \ldots \ldots \ldots \ldots$. & $1-5 \mathrm{Gyr}$ & $0.5-8 \mathrm{Gyr}$ \\
Age for $z=1.4 \ldots \ldots \ldots \ldots$ & $3 \mathrm{Myr}-3 \mathrm{Gyr}$ & $1 \mathrm{Myr}-6 \mathrm{Gyr}$ \\
Metallicity $\left(Z \odot Z_{\odot}\right) \ldots \ldots \ldots$. & $0.2,0.4,1.0,2.5,5$ & $0.2,0.4,1.0,2.5,5$ \\
\hline
\end{tabular}

${ }^{1}$ Range from which synthetic colors of the galaxy were generated.

${ }^{2}$ Range in the model parameters for the data-model comparison.

see Fioc \& Rocca-Volmerange 1997). Although the theoretical isochrones in both models come mainly from the Padua group (Bressan et al. 1993), there are fundamental differences in post-main-sequence evolutionary stages between the two set of models, including in the early, thermally pulsating, and post-asymptotic giant branch (post-AGB) phases. It is noteworthy that the differences in these evolutionary stages are responsible for the most serious uncertainties in the stellar population modeling (see Charlot et al. 1996). In Figure 1 we show the predictions of these two models for simple stellar population (SSP) and continuous star formation galaxies with solar metallicity. For the sake of comparison we have also included the predictions of the Starburst 99 models (Leitherer et al. 1999) at ages younger than 0.1 Gyr.

\section{ANALYSIS \\ 3.1. Galaxy Colors}

Once the sample was generated we determined the luminosity (per solar mass) in the different bands and the colors for each individual galaxy in the sample by using the predictions of the corresponding evolutionary synthesis models. We adopted for all the models the same Salpeter initial mass function (IMF) with $M_{\text {low }}=0.1 M_{\odot}$ and $M_{\text {up }}=100 M_{\odot}$.

The bands considered in this work include the JohnsonCousins $\left(U B V R_{C} I_{C}\right)$ and Sloan Digital Sky Survey $u^{\prime} g^{\prime} r^{\prime} i^{\prime} z^{\prime}$ (Fukugita et al. 1996) optical bands, the $J H K$ " near-infrared" bands, and the GALEX "near-ultraviolet" (NUV; 1800-3000 $\AA$ ) and far-ultraviolet bands (FUV; 1350-1800 $\AA$; Doliber et al. 2000). Because of the small differences expected between the standard $K$ band and the $K_{s}$ and $\mathrm{K}^{\prime}$ bands, we decided to include only the standard $K$ bands in our realizations. In this sense, although the 2MASS survey has been carried out using $K_{s}$-band imaging data, we will refer hereafter to the 2MASS data set when dealing with the standard $J H K$ bands.

The colors obtained for each stellar population were then reddened (and the apparent mass-to-light ratios increased) by using the corresponding $E(B-V)$ values and adopting the parameterization of the Galactic extinction law of Cardelli, Clayton, \& Mathis (1989) for a total-to-selective extinction ratio $R_{V}=3.1$. However, since this parameterization is valid down to only $1000 \mathrm{~A}$ and given that some of the bands selected for this work cover regions of galaxy spectra well below the Lyman limit (for $z=0.7$ and $z=1.4$ ), we extended the extinction law to the far-UV by using the $A_{\lambda} / A_{V}$ ratios given by Mathis (1990; see also 

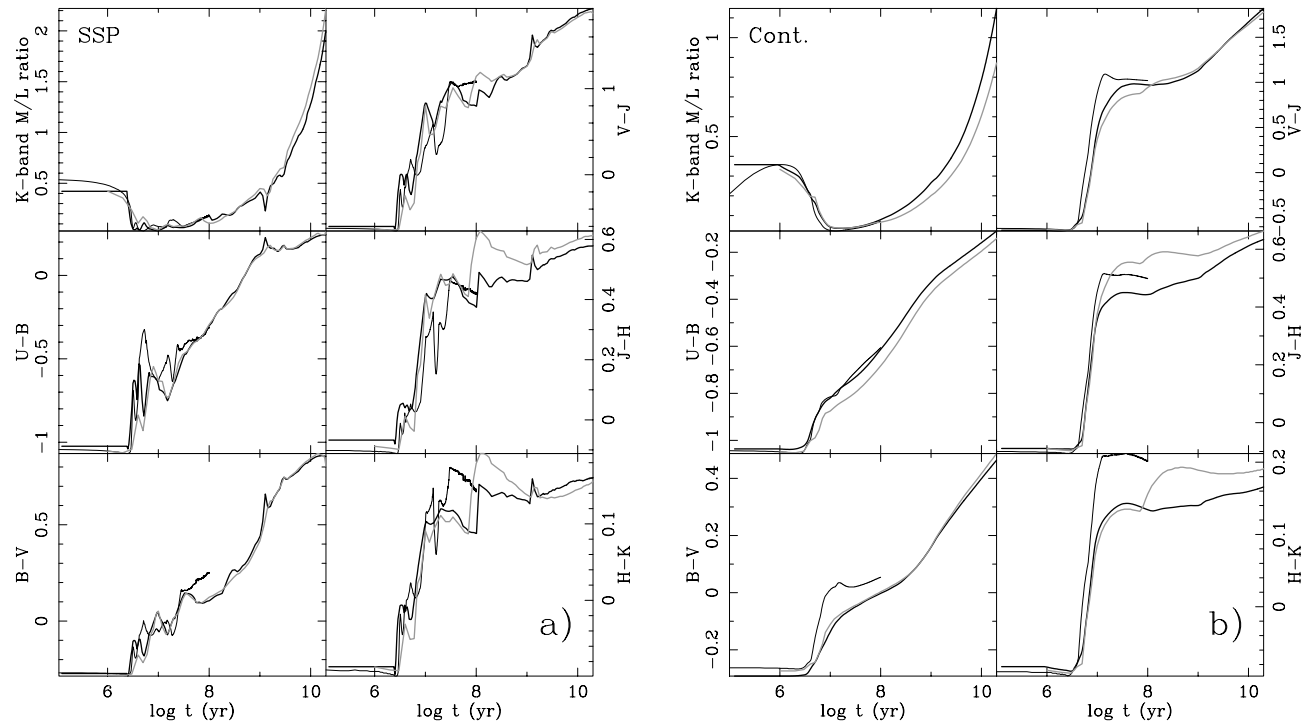

FIG. 1.-Comparison of the predictions of the Bruzual \& Charlot (2002; thick lines), the PÉGASE (Version 2.0; see Fioc \& Rocca-Volmerange 1997; thick gray lines), and Starburst 99 (Leitherer et al. 1999; thin lines) models for a solar metallicity galaxy with $(a)$ instantaneous (SSP) and (b) continuous star formation.

Martin \& Rouleau, 1989). The $A_{\lambda} / A_{V}$ mean values for each band (its respective redshifted rest-frame wavelength) were then computed by convolving the filter response functions with the adopted extinction curve.

Using the number of ionizing Lyman photons predicted by the evolutionary synthesis models, we also computed the contribution of the nebular continuum and most intense gas emission lines in all the bands considered. We assumed that $85 \%$ of the photons with $\lambda<912 \AA$ effectively ionize the surrounding gas, but a $15 \%$ fraction would be observed at the far-ultraviolet or absorbed by dust (Leitherer et al. 1995; Dove, Shull, \& Ferrara 2000; GIL00).

Most of the $85 \%$ of the far-UV photons absorbed by the surrounding gas in the $\mathrm{H}$ II region are reprocessed as nebular continuum and emission lines in the optical and NIR. However, a very small fraction of these photons can be reemitted as free-free radiation in the far-UV. In our case, we assumed that these secondary far-UV photons are not absorbed by neutral gas again, but rather are absorbed by dust or escape from the galaxy. To determine the nebular continuum contribution to all the bands, we have used the emission and recombination coefficients given by Ferland (1980) for the near-UV, optical, and NIR for $T_{e}=10^{4} \mathrm{~K}$. For the far-UV free-free radiation we have assumed a constant Gaunt factor $\bar{g}_{f f}=1.1$ (see Karzas \& Latter 1961) in the range $500-912 \AA$ for a gas with $T_{e}=10^{4} \mathrm{~K}$.

With regard to the gas emission lines we have assumed the relation between the number of Lyman photons and $\mathrm{H} \alpha$ luminosity given by Brocklehurst (1971) and the theoretical hydrogen line ratios expected for a low-density gas $\left(n_{e}=10^{2} \mathrm{~cm}^{-3}\right)$ with $T_{e}=10^{4} \mathrm{~K}$ in case B recombination (Osterbrock 1989). We considered the contribution of the most intense forbidden lines ([O $\mathrm{II}] \lambda \lambda 3726,3729$, [O III] $\lambda \lambda 4959,5007,[\mathrm{~N} \mathrm{II}] \lambda \lambda 6548,6583$, and [S II] $\lambda \lambda 6717,6731)$ by adopting the mean line ratios measured by Gallego et al. (1996) for the Universidad Complutense de Madrid sample of local star-forming galaxies (Zamorano et al. 1994, 1996). Both the nebular continuum and the emission-line luminosities were corrected for extinction by assuming the relation given by Calzetti, Kinney, \& Storchi-Bergmann (1996; see also Calzetti 1997; Storchi-Bergmann, Calzetti, \& Kinney 1994) between the gas and the stellar continuum reddening associated with the young stellar population: $E(B-V)_{\text {stl }}$ $=0.44 E(B-V)_{\text {gas }}$.

Following this procedure we obtain all the input information concerning the actual properties, colors, and mass-tolight ratios for the galaxies in the sample. In the next section we discuss our recovery of these input properties, starting from the observed galaxy colors, and their corresponding measuring errors.

\subsection{Sets of Colors}

Grouping the colors deduced for these galaxies in different sets and comparing them with the evolutionary synthesis models allow us to explore systematic errors and determine the set of observables that result in minimum differences between the actual (input) galaxy properties and the derived (output) properties. This comparison is done using a combination of Monte Carlo simulations, a maximum likelihood estimator, and a principal component analysis algorithm.

The number of different combinations of colors that can be constructed considering a total of 10 potential bands from the UV to the near-infrared is $\sum_{r=2}^{10} 10 ! /[r !(10-r) !]$ $=1013$. For this study we have selected only the 10 sets shown in Table 2. The detailed comparison of the results obtained for all these sets provides enough information about the relevance of the different bands, observing errors, etc., for a precise determination of the galaxy properties.

\subsection{Comparison Procedure}

Once the colors of the galaxy sample had been obtained and grouped in sets we then perturbed the "observed " magnitudes by applying random observing errors. To simplify the problem we studied three cases, corresponding to three different $1 \sigma$ errors in the colors, $0.03,0.07$, and $0.10 \mathrm{mag}$, and considered only the case for which these errors are the same in all the colors. 
TABLE 2

Definition of the Different Combinations of Filters Analyzed

\begin{tabular}{|c|c|c|}
\hline Bands & $\begin{array}{l}\text { Number } \\
\text { of Bands }\end{array}$ & Alias \\
\hline$B, V, R_{C}, I_{C} \ldots \ldots \ldots \ldots \ldots \ldots$ & 4 & $B V R I$ \\
\hline$U, B, V, R_{C}, I_{C} \ldots \ldots \ldots \ldots \ldots$ & 5 & $U+B V R I$ \\
\hline$U, B, V, R_{C}, I_{C}, K \ldots \ldots \ldots$ & 6 & $U+B V R I+K$ \\
\hline$U, B, V, R_{C}, I_{C}, J, H, K \ldots$ & 8 & $U+B V R I+J H K$ \\
\hline$B, V, R_{C}, I_{C}, J, H, K \ldots \ldots \ldots$ & 7 & $B V R I+J H K$ \\
\hline$B, V, R_{C}, I_{C}, K \ldots \ldots \ldots \ldots \ldots$ & 5 & $B V R I+K$ \\
\hline$U, V, I_{C}, J, K \ldots \ldots \ldots \ldots \ldots$ & 5 & UVIJK \\
\hline$u^{\prime}, g^{\prime}, r^{\prime}, i^{\prime}, z^{\prime} \ldots \ldots \ldots \ldots \ldots \ldots \ldots$ & 5 & SDSS \\
\hline$u^{\prime}, g^{\prime}, r^{\prime}, i^{\prime}, z^{\prime}, J, H, K \ldots \ldots$ & 8 & SDSS +2 MASS \\
\hline $\begin{array}{l}\text { FUV, NUV, } u^{\prime}, g^{\prime}, r^{\prime}, \\
\quad i^{\prime}, z^{\prime}, J, H, K \ldots \ldots \ldots \ldots \ldots . . .\end{array}$ & 10 & GALEX + SDSS +2 MASS \\
\hline
\end{tabular}

To compute the effects of these observing errors in the galaxy properties to be derived we used a Gaussian distribution of errors for all bands generated by using a Monte Carlo simulation method. The colors derived for each test particle were then compared with the evolutionary synthesis models by using a maximum likelihood estimator, $L$. The expression for this estimator is

$$
L=\prod_{n=1}^{N} \frac{1}{\sqrt{2 \pi} \Delta C_{n}} \exp \left[-\frac{\left(c_{n}-C_{n}\right)^{2}}{2 \Delta C_{n}^{2}}\right],
$$

where the $C_{n}$ are the colors derived, the $c_{n}$ are those predicted by the evolutionary synthesis models, and $N$ is the total number of colors available within each set (see, e. g., Abraham et al. 1999). Because the same level of error was assumed for all the bands, maximizing this expression is equivalent to computing the minimum $\chi^{2}$; therefore in this case we could estimate the confidence levels in the galaxy properties via Avni's approximation (Avni 1976), as has been done by Bolzonella et al. (2000), instead of using numerical simulations. However, the considerations that lead to this estimation procedure apply only asymptotically, being applicable when the $\chi^{2}$ estimator covariance matrix can be replaced by its linear approximation in the vicinity of the best-fitting set of parameters. Although these conditions could be fulfilled in our case (Perez-González et al. 2002), we have decided to use numerical simulations to be able to derive the degeneracies between the different galaxy properties.

The ranges in the evolutionary synthesis models parameters in which the data-model comparison was performed are shown in Table 1. To avoid introducing a constraint bias in the derived properties, the ranges for these comparisons were chosen to be significantly wider than those for which the galaxy sample was generated.

Once the expression $L$ is maximized for a significant number of Monte Carlo test particles (we used a total of 200) we obtained the distribution of physical properties associated with the probability distribution of the galaxy colors. In Figure 2 we show the results obtained for a nearby galaxy with an exponential star formation history with timescale $\tau=4.5 \mathrm{Gyr}$, solar metallicity, age $5.0 \mathrm{Gyr}$, and $E(B-V)$ $0.08 \mathrm{mag}$, for observing errors in the colors $(a) 0.10,(b) 0.07$, and (c) $0.03 \mathrm{mag}$. In this case the set of colors used included the GALEX, SDSS, and 2MASS bands (see Table 2).
These figures illustrate the strong degeneracy between the different galaxy properties even for relatively small measurement errors. We have therefore performed a quantitative analysis of these degeneracies by using a principal component analysis (PCA) on the space of galaxy properties by solving the eigenvector equation for the test particle correlation matrix of each galaxy in the sample (see Morrison 1976). This analysis gives the direction in the space of galaxy properties along which the 200 solutions obtained for each individual galaxy are mainly oriented, constituting the best estimator of the degeneracy between these properties.

In summary, this procedure provides us with (1) the mean derived properties, (2) the $1 \sigma$ errors, (3) the orientation in the space of solutions of the principal component (PCA1), and (4) the input (i.e., actual) properties of all galaxies in the sample.

\section{RESULTS}

Once these quantities had been derived we computed the mean differences between the output and input values, along with the mean $1 \sigma$ spread, at fixed intervals in the input properties. The bins used were $0.5 \mathrm{Gyr}, 0.025 \mathrm{dex}$, and 0.025 mag in the formation timescale, age, and reddening, respectively. Mean differences and $1 \sigma$ values in the stellar metallicity were computed for each of the input values considered.

In Figure 3 we show the results obtained before and after computing the mean differences and $1 \sigma$ errors for a subsample of 500 nearby galaxies with errors in the colors of 0.07 mag and $U+B V R I+J H K$ data available. Because of the relevance of the $K$-band luminosities to derive stellar masses in nearby (Aragón-Salamanca et al. 1993; GIL00) and intermediate-redshift galaxies (see Brinchmann \& Ellis 2000 and references therein), the mean differences between the derived and input $K$-band mass-to-light ratios were also computed. Mean uncertainties for all the sets of observables, redshifts, and observing errors considered are summarized in Table 3.

In addition, we studied the degeneracies between the galaxy physical properties by analyzing the distribution of the unitary PCA1 vector components. In Figure $4 a$ we show the frequency histograms obtained for the sample of high-redshift galaxies, assuming an error in the colors of $0.07 \mathrm{mag}$, and the SDSS, SDSS + 2MASS, and GALEX SDSS + 2MASS sets. Note that the PCA1 vector points toward the direction where the largest fraction of the galaxy properties' variance occurs. In this sense, a PCA1 vector with components $\left(u_{\log t}, \quad u_{E(B-V)}, \quad u_{\log Z / Z_{\odot}}, \quad u_{\tau}\right)$ $=(+0.707,-0.707,0,0)$, say, implies the existence of a degeneracy between age and reddening in the sense that younger obscured stellar populations have colors that are indistinguishable from older but less extincted ones. In this case, no age-metallicity or age-timescale degeneracies would be present. However, the behavior described above could also result in a PCA1 vector with components $\left(u_{\log t}, u_{E(B-V)}, u_{\log Z / Z_{\odot}}, u_{\tau}\right)=(-0.707,+0.707,0,0)$. That, however, would appear in a different position in the frequency histogram shown in Figure $4 a$. Thus, to reduce this sign ambiguity when interpreting our results we forced the $u_{\log t}$ component to be positive, changing the sign of all the vector components if $u_{\log t}$ was negative. Finally, to quantitatively determine the dominant degeneracy for each individual galaxy in our sample we have defined the angle 

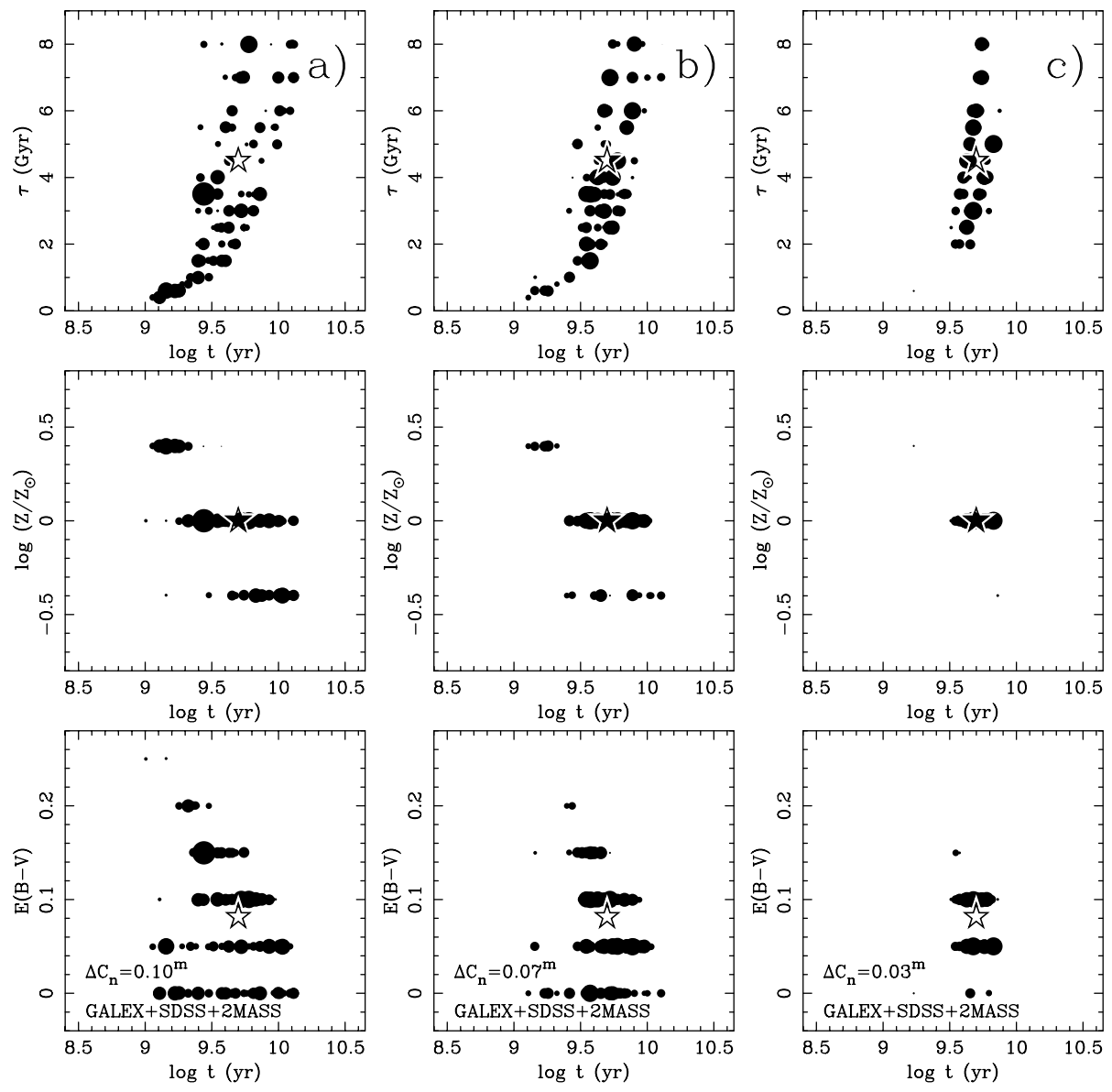

FIG. 2.-Top, Distribution of solutions associated with the 200 Monte Carlo simulations in timescale-age space for a nearby galaxy with formation timescale $4.5 \mathrm{Gyr}$, age $5 \mathrm{Gyr}$, dust extinction $E(B-V)=0.08 \mathrm{mag}$, and solar metallicity; middle, same as (top) but for age-metallicity space; bottom, same as (top) but for age-extinction space. Observing errors are $(a) 0.10,(b) 0.07$, and $(c) 0.03$ mag, respectively. The input properties of the galaxy are marked with a star. This comparison was performed for the GALEX + SDSS + 2MASS color set. The size of each point is proportional to the value of the maximum likelihood estimator, $L$, for the corresponding Monte Carlo simulation. Degeneracies between the galaxy properties are evidenced by correlations in the distribution of points.

$\theta_{i, j}$ as that satisfying

$$
\cos \theta_{i, j}=\operatorname{SIGN}\left(\frac{u_{i}}{u_{j}}\right) \sqrt{u_{i}^{2}+u_{j}^{2}}
$$

where $u_{i}$ and $u_{i}$ are the $i$ th and $j$ th components of the PCA1 vector. The angle $\theta_{i, j}$ simultaneously provides a measure of the angle between the PCA1 vector and the $(i, j)$ plane of physical properties and the sign of the degeneracy between the $i$ and $j$ properties. Thus, if $\left|\cos \theta_{i, j}\right| \simeq 1$ the degeneracy between the $i$ and $j$ properties would dominate the total degeneracy. Moreover, if $\cos \theta_{i, j}>0$ an increase in both the $i$ and $j$ properties would lead to similar observational properties, while if $\cos \theta_{i, j}<0$ the value of one of the properties should decrease. In Figure $4 b$ we show the distribution of $\cos \theta_{i, j}$ as a function of the age for the high-redshift sample, assuming an error in the colors of $0.07 \mathrm{mag}$ and the GALEX + SDSS + 2MASS set available.

In this section we will describe the results obtained from the analysis of the distributions shown in Figures 3 and 4 for the different redshifts, observing errors, and bandpass combinations considered.

\subsection{Nearby Galaxies \\ 4.1.1. Formation Timescale}

With regard to the formation timescale in nearby galaxies, Figure $5 a$ indicates that, even for relatively small observing errors, its uncertainty is very high (see also Table 3 ) and shows a strong dependence on the value of the formation timescale itself. The larger uncertainty in the formation timescale for larger values of this quantity is mainly due to the small sensitivity of the optical-NIR colors of stellar populations with ages $t \ll \tau$ to changes in its formation timescale. The use of $U$-band data significantly reduces this uncertainty, probably because of the high sensitivity of this band to the presence of recent star formation, which allows us to rule out instantaneous-burst solutions when recent star formation associated with larger $\tau$ values has effectively taken place. The use of NIR data, however, does not provide relevant information about the formation timescale of the stellar population. Moreover, the reduction in the uncertainties of the different galaxy properties achieved by using $J H K$ data compared with that obtained by using exclusively $K$-band data is very small (see Table 3 ). As we will show in $\S \S 4.2$ and 4.3 this is not the case for the intermediate- and 

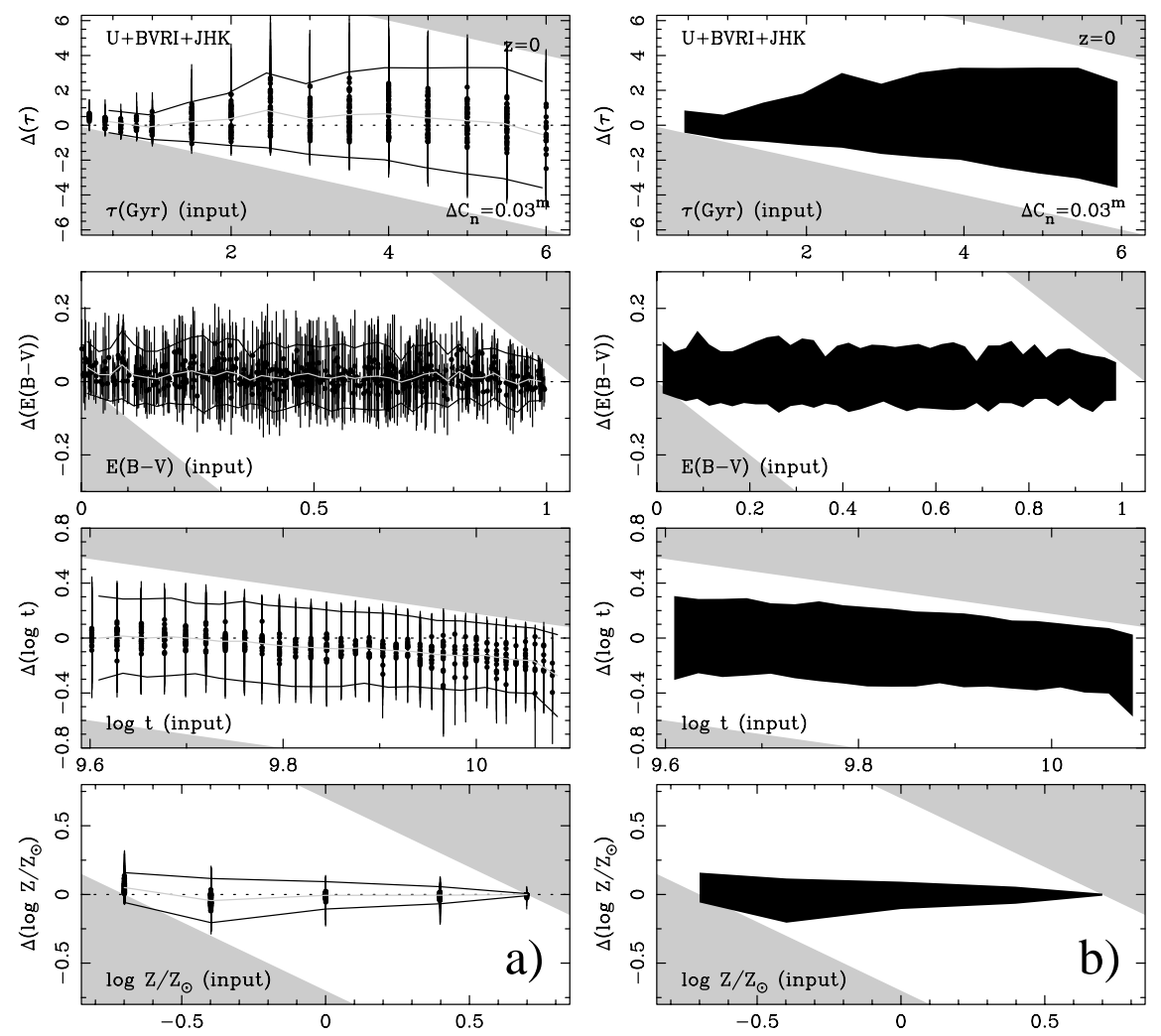

FIG. 3.-Uncertainties in the derived properties as a function of the properties themselves for a subsample of 500 nearby galaxies and the $U+B V R I+J H K$ set of observables. (a) Mean differences between the derived and the input properties computed in intervals of $0.5 \mathrm{Gyr}$ in formation timescale, 0.025 mag in color excess, and 0.05 dex in age represented by a light gray line. Mean $\pm 1 \sigma$ values for the derived properties are delimited by two dark gray lines. (b) Region defined by the mean $\pm 1 \sigma$ lines. Gray shaded areas represent the regions not covered by our comparison procedure.

high-redshift galaxies, in which these bands now cover the redshifted optical spectrum. Finally, in the same way as in the $U$ band, the use of UV data provides an additional reduction in the formation timescale. As is clearly seen in Figure 2, for a particular galaxy the formation timescale is mainly degenerate with the age of the stellar population, in the sense that within the observing errors assumed an increase in the formation timescale accompanied by an increase in the age can result in similar UV-optical-NIR colors. Because of this age-timescale degeneracy, part of the reduction in the timescale uncertainty obtained by the use of UV data can be explained by the significant reduction in the age uncertainty achieved by including UV data (see below).

\subsubsection{Age}

With respect to the age determination in nearby galaxies $(z=0)$, Figure $5 a$ also shows that a significant reduction in the age uncertainty is achieved by including NIR data. It is important to note that the use of additional NIR data results in the same dominant degeneracy as if only optical data are used (see below), but the range of physical properties in which this degeneracy takes place is significantly smaller. The most significant improvement in the age determination, however, is obtained when UV data are available. This is mainly due to the emission arising from post-AGB stars in low-metallicity populations and at ages younger than $10 \mathrm{Gyr}$ and to the UV upturn in high-metallicity evolved $(t>10 \mathrm{Gyr})$ stellar populations that result in highly peculiar UV-optical colors. However, the uncertainty in the modeling of post-AGB stars (Charlot et al. 1996) and the low-mass core-helium-burning horizontal branch (HB) and evolved HB stars that lead to the UV upturn (Yi et al. 1997) introduce additional errors in the UV-optical colors during the data-model comparison. Charlot el al. (1996) estimated by using two different theoretical prescriptions that the uncertainty in only the post-AGB phase modeling could result in differences of about $1 \mathrm{mag}$ in the UV-optical colors of a several gigayear-old stellar population. Moreover, although we assumed the same observing errors for all the bands, the faint UV emission of evolved stellar populations is expected to result in very large observing errors in the UV-optical colors. Therefore, while the stellar evolution of these stars is not well understood the age determination in old stellar populations should not rely on UV data.

Along with the formation timescale, the age of the stellar population in nearby galaxies is mainly degenerate with the dust extinction, in the sense that older stellar populations with low dust content have colors similar to highly extincted younger stellar populations. Although in the case of very old stellar populations the age-extinction degeneracy also competes with the age-metallicity degeneracy (see Worthey 1994), the age-extinction degeneracy is still dominant in this range for all the bandpass combinations and observing errors considered in this work. It could be argued that the strong discretization of the metallicity in our models could be responsible for the relatively weak age-metallicity degeneration derived. However, the fact that this behavior is 
TABLE 3

Mean $1 \sigma$ Uncertainties in the Derived Properties

\begin{tabular}{|c|c|c|c|c|c|c|c|c|c|}
\hline \multirow[b]{2}{*}{ Property } & \multicolumn{3}{|c|}{$z=0$} & \multicolumn{3}{|c|}{$z=0.7$} & \multicolumn{3}{|c|}{$z=1.4$} \\
\hline & $\begin{array}{c}\Delta C_{n}=0.03 \\
(\mathrm{mag})\end{array}$ & $\begin{array}{c}\Delta C_{n}=0.07 \\
\quad(\mathrm{mag})\end{array}$ & $\begin{array}{c}\Delta C_{n}=0.10 \\
(\mathrm{mag})\end{array}$ & $\begin{array}{c}\Delta C_{n}=0.03 \\
(\mathrm{mag})\end{array}$ & $\begin{array}{c}\Delta C_{n}=0.07 \\
\quad(\mathrm{mag})\end{array}$ & $\begin{array}{c}\Delta C_{n}=0.10 \\
\quad(\mathrm{mag})\end{array}$ & $\begin{array}{c}\Delta C_{n}=0.03 \\
\quad(\mathrm{mag})\end{array}$ & $\begin{array}{c}\Delta C_{n}=0.07 \\
\quad(\mathrm{mag})\end{array}$ & $\begin{array}{c}\Delta C_{n}=0.10 \\
(\mathrm{mag})\end{array}$ \\
\hline \multicolumn{10}{|c|}{$B V R I$} \\
\hline$E(B-V)(\mathrm{mag}) \ldots \ldots \ldots \ldots$ & 0.16 & 0.17 & 0.17 & 0.11 & 0.18 & 0.20 & 0.02 & 0.04 & 0.06 \\
\hline$\tau(\mathrm{Gyr}) \ldots \ldots \ldots \ldots \ldots \ldots$ & 2.50 & 2.87 & 3.07 & 2.89 & 3.10 & 3.32 & 2.91 & 3.33 & 3.42 \\
\hline $\log t(\operatorname{dex}) \ldots \ldots$ & 0.36 & 0.37 & 0.38 & 0.23 & 0.31 & 0.35 & 0.45 & 0.71 & 0.84 \\
\hline $\log Z(\operatorname{dex}) \ldots \ldots \ldots \ldots$ & 0.33 & 0.33 & 0.33 & 0.44 & 0.46 & 0.45 & 0.37 & 0.45 & 0.48 \\
\hline$(M / L)_{K}\left(M_{\odot} / L_{K, \odot}\right) \ldots \ldots$ & 0.32 & 0.41 & 0.47 & 0.06 & 0.08 & 0.09 & 0.03 & 0.05 & 0.06 \\
\hline \multicolumn{10}{|c|}{$U+B V R I$} \\
\hline$E(B-V)(\mathrm{mag}) \ldots \ldots \ldots \ldots$ & 0.14 & 0.18 & 0.19 & 0.02 & 0.04 & 0.07 & 0.02 & 0.04 & 0.06 \\
\hline$\tau(\mathrm{Gyr})$ & 2.27 & 2.69 & 3.00 & 3.10 & 3.10 & 3.11 & 3.11 & 3.34 & 3.33 \\
\hline $\log t(\operatorname{dex}) \ldots \ldots \ldots \ldots$ & 0.34 & 0.37 & 0.38 & 0.23 & 0.30 & 0.34 & 0.45 & 0.68 & 0.81 \\
\hline $\log Z(\operatorname{dex}) \ldots \ldots \ldots \ldots \ldots \ldots$ & 0.32 & 0.41 & 0.45 & 0.38 & 0.51 & 0.54 & 0.29 & 0.37 & 0.38 \\
\hline$(M / L)_{K}\left(M_{\odot} / L_{K, \odot}\right) \ldots \ldots$ & 0.32 & 0.41 & 0.47 & 0.05 & 0.08 & 0.09 & 0.03 & 0.05 & 0.06 \\
\hline \multicolumn{10}{|c|}{$U+B V R I+K$} \\
\hline$E(B-V)(\mathrm{mag}) \ldots \ldots \ldots \ldots$ & 0.09 & 0.14 & 0.17 & 0.02 & 0.04 & 0.05 & 0.02 & 0.04 & 0.05 \\
\hline$\tau(\mathrm{Gyr}) \ldots \ldots \ldots \ldots \ldots \ldots$ & 2.19 & 2.75 & 3.03 & 2.29 & 2.95 & 3.19 & 2.79 & 3.00 & 3.08 \\
\hline $\log t(\operatorname{dex}) \ldots \ldots \ldots \ldots \ldots$ & 0.28 & 0.35 & 0.38 & 0.13 & 0.21 & 0.24 & 0.20 & 0.28 & 0.33 \\
\hline $\log Z(\operatorname{dex}) \ldots \ldots \ldots \ldots \ldots$ & 0.11 & 0.20 & 0.26 & 0.15 & 0.22 & 0.26 & 0.17 & 0.29 & 0.34 \\
\hline$(M / L)_{K}\left(M_{\odot} / L_{K, \odot}\right) \ldots \ldots$ & 0.26 & 0.33 & 0.35 & 0.03 & 0.06 & 0.06 & 0.01 & 0.02 & 0.03 \\
\hline \multicolumn{10}{|c|}{$U+B V R I+J H K$} \\
\hline$E(B-V)(\mathrm{mag}) \ldots \ldots \ldots \ldots$ & 0.08 & 0.14 & 0.17 & 0.02 & 0.03 & 0.05 & 0.02 & 0.03 & 0.04 \\
\hline$\tau(\mathrm{Gyr})$ & 2.12 & 2.70 & 2.96 & 2.35 & 3.07 & 3.35 & 3.19 & 3.44 & 3.44 \\
\hline $\log t(\mathrm{dex}) \ldots \ldots \ldots \ldots \ldots$ & 0.26 & 0.36 & 0.39 & 0.09 & 0.17 & 0.22 & 0.12 & 0.23 & 0.29 \\
\hline $\log Z(\operatorname{dex}) \ldots \ldots \ldots \ldots \ldots$ & 0.09 & 0.20 & 0.26 & 0.10 & 0.17 & 0.22 & 0.06 & 0.17 & 0.23 \\
\hline$(M / L)_{K}\left(M_{\odot} / L_{K, \odot}\right) \ldots \ldots$ & 0.24 & 0.35 & 0.37 & 0.02 & 0.04 & 0.05 & 0.01 & 0.01 & 0.02 \\
\hline \multicolumn{10}{|c|}{$B V R I+J H K$} \\
\hline$E(B-V)(\mathrm{mag}) \ldots \ldots \ldots \ldots$ & 0.10 & 0.17 & 0.18 & 0.04 & 0.10 & 0.13 & 0.02 & 0.03 & 0.05 \\
\hline$\tau(\mathrm{Gyr}) \ldots \ldots \ldots \ldots$ & 2.53 & 2.97 & 3.14 & 2.38 & 3.08 & 3.30 & 3.22 & 3.47 & 3.45 \\
\hline $\log t(\operatorname{dex}) \ldots \ldots \ldots \ldots \ldots \ldots$ & 0.32 & 0.38 & 0.40 & 0.12 & 0.22 & 0.28 & 0.13 & 0.24 & 0.29 \\
\hline $\log Z(\operatorname{dex}) \ldots \ldots \ldots \ldots \ldots \ldots$ & 0.13 & 0.23 & 0.28 & 0.11 & 0.20 & 0.25 & 0.07 & 0.19 & 0.25 \\
\hline$(M / L)_{K}\left(M_{\odot} / L_{K, \odot}\right) \ldots \ldots$ & 0.29 & 0.37 & 0.39 & 0.03 & 0.05 & 0.06 & 0.01 & 0.02 & 0.02 \\
\hline \multicolumn{10}{|c|}{$B V R I+K$} \\
\hline$E(B-V)(\mathrm{mag}) \ldots \ldots \ldots \ldots$ & 0.12 & 0.17 & 0.18 & 0.08 & 0.14 & 0.16 & 0.02 & 0.04 & 0.05 \\
\hline$\tau(\mathrm{Gyr}) \ldots \ldots \ldots \ldots \ldots \ldots$ & 2.67 & 3.15 & 3.35 & 2.32 & 2.88 & 3.06 & 2.72 & 2.92 & 3.03 \\
\hline $\log t(\operatorname{dex})$ & 0.34 & 0.37 & 0.38 & 0.16 & 0.25 & 0.30 & 0.22 & 0.30 & 0.34 \\
\hline $\log Z(\operatorname{dex}) \ldots \ldots \ldots \ldots$ & 0.15 & 0.23 & 0.28 & 0.20 & 0.29 & 0.31 & 0.26 & 0.39 & 0.44 \\
\hline$(M / L)_{K}\left(M_{\odot} / L_{K, \odot}\right) \ldots \ldots$ & 0.30 & 0.34 & 0.36 & 0.05 & 0.08 & 0.09 & 0.02 & 0.03 & 0.03 \\
\hline
\end{tabular}


TABLE 3-Continued

\begin{tabular}{|c|c|c|c|c|c|c|c|c|c|}
\hline \multirow[b]{2}{*}{ Property } & \multicolumn{3}{|c|}{$z=0$} & \multicolumn{3}{|c|}{$z=0.7$} & \multicolumn{3}{|c|}{$z=1.4$} \\
\hline & $\begin{array}{c}\Delta C_{n}=0.03 \\
(\mathrm{mag})\end{array}$ & $\begin{array}{c}\Delta C_{n}=0.07 \\
\quad(\mathrm{mag})\end{array}$ & $\begin{array}{c}\Delta C_{n}=0.10 \\
(\mathrm{mag})\end{array}$ & $\begin{array}{c}\Delta C_{n}=0.03 \\
\quad(\mathrm{mag})\end{array}$ & $\begin{array}{c}\Delta C_{n}=0.07 \\
\quad(\mathrm{mag})\end{array}$ & $\begin{array}{c}\Delta C_{n}=0.10 \\
(\mathrm{mag})\end{array}$ & $\begin{array}{c}\Delta C_{n}=0.03 \\
\quad(\mathrm{mag})\end{array}$ & $\begin{array}{c}\Delta C_{n}=0.07 \\
\quad(\mathrm{mag})\end{array}$ & $\begin{array}{c}\Delta C_{n}=0.10 \\
\quad(\mathrm{mag})\end{array}$ \\
\hline \multicolumn{10}{|c|}{ UVIJK } \\
\hline$E(B-V)(\mathrm{mag}) \ldots \ldots \ldots \ldots$ & 0.10 & 0.16 & 0.19 & 0.02 & 0.04 & 0.07 & 0.02 & 0.03 & 0.05 \\
\hline$\tau(\mathrm{Gyr})$ & 2.33 & 2.87 & 3.14 & 2.54 & 3.21 & 3.49 & 3.14 & 3.41 & 3.42 \\
\hline $\log t(\operatorname{dex})$ & 0.30 & 0.36 & 0.38 & 0.11 & 0.18 & 0.23 & 0.16 & 0.27 & 0.33 \\
\hline $\log Z(\operatorname{dex}) \ldots \ldots \ldots \ldots$ & 0.13 & 0.24 & 0.29 & 0.11 & 0.18 & 0.23 & 0.08 & 0.19 & 0.24 \\
\hline$(M / L)_{K}\left(M_{\odot} / L_{K, \odot}\right) \ldots \ldots$ & 0.29 & 0.36 & 0.38 & 0.02 & 0.04 & 0.05 & 0.01 & 0.02 & 0.02 \\
\hline \multicolumn{10}{|c|}{ SDSS } \\
\hline$E(B-V)(\mathrm{mag}) \ldots \ldots \ldots \ldots$ & 0.10 & 0.14 & 0.17 & 0.02 & 0.06 & 0.09 & 0.03 & 0.05 & 0.07 \\
\hline$\tau(\mathrm{Gyr})$ & 2.28 & 2.73 & 2.93 & 2.93 & 3.11 & 3.23 & 2.94 & 3.05 & 2.97 \\
\hline $\log t(\mathrm{dex})$ & 0.31 & 0.39 & 0.41 & 0.18 & 0.26 & 0.31 & 0.39 & 0.61 & 0.74 \\
\hline $\log Z(\operatorname{dex}) \ldots \ldots \ldots \ldots \ldots \ldots$ & 0.21 & 0.31 & 0.35 & 0.40 & 0.44 & 0.45 & 0.25 & 0.32 & 0.37 \\
\hline$(M / L)_{K}\left(M_{\odot} / L_{K, \odot}\right) \ldots \ldots$ & 0.30 & 0.38 & 0.40 & 0.04 & 0.06 & 0.08 & 0.02 & 0.04 & 0.05 \\
\hline \multicolumn{10}{|c|}{ SDSS + 2MASS } \\
\hline$E(B-V)(\mathrm{mag}) \ldots \ldots \ldots \ldots$ & 0.07 & 0.14 & 0.16 & 0.02 & 0.04 & 0.05 & 0.02 & 0.03 & 0.04 \\
\hline$\tau(\mathrm{Gyr})$ & 2.06 & 2.65 & 2.89 & 2.36 & 3.07 & 3.34 & 3.20 & 3.41 & 3.39 \\
\hline $\log t(\mathrm{dex}) \ldots \ldots \ldots \ldots$ & 0.25 & 0.36 & 0.39 & 0.09 & 0.16 & 0.21 & 0.12 & 0.21 & 0.26 \\
\hline $\log Z(\operatorname{dex}) \ldots \ldots \ldots \ldots \ldots$ & 0.08 & 0.19 & 0.25 & 0.10 & 0.16 & 0.21 & 0.08 & 0.17 & 0.23 \\
\hline$(M / L)_{K}\left(M_{\odot} / L_{K, \odot}\right) \ldots \ldots$ & 0.24 & 0.35 & 0.37 & 0.02 & 0.04 & 0.05 & 0.01 & 0.01 & 0.02 \\
\hline \multicolumn{10}{|c|}{ GALEX + SDSS + 2MASS } \\
\hline$E(B-V)(\mathrm{mag}) \ldots \ldots \ldots \ldots$ & 0.04 & 0.09 & 0.12 & 0.01 & 0.02 & 0.02 & 0.01 & 0.02 & 0.03 \\
\hline$\tau(\mathrm{Gyr})$ & 1.51 & 2.26 & 2.60 & 2.01 & 2.93 & 3.26 & 2.92 & 3.63 & 3.67 \\
\hline $\log t(\operatorname{dex}) \ldots \ldots \ldots \ldots$ & 0.10 & 0.19 & 0.24 & 0.08 & 0.13 & 0.17 & 0.09 & 0.16 & 0.21 \\
\hline $\log Z(\operatorname{dex}) \ldots \ldots \ldots \ldots \ldots$ & 0.05 & 0.16 & 0.22 & 0.11 & 0.16 & 0.20 & 0.03 & 0.08 & 0.14 \\
\hline$(M / L)_{K}\left(M_{\odot} / L_{K, \odot}\right) \ldots \ldots$ & 0.10 & 0.23 & 0.28 & 0.02 & 0.03 & 0.04 & 0.01 & 0.01 & 0.01 \\
\hline
\end{tabular}



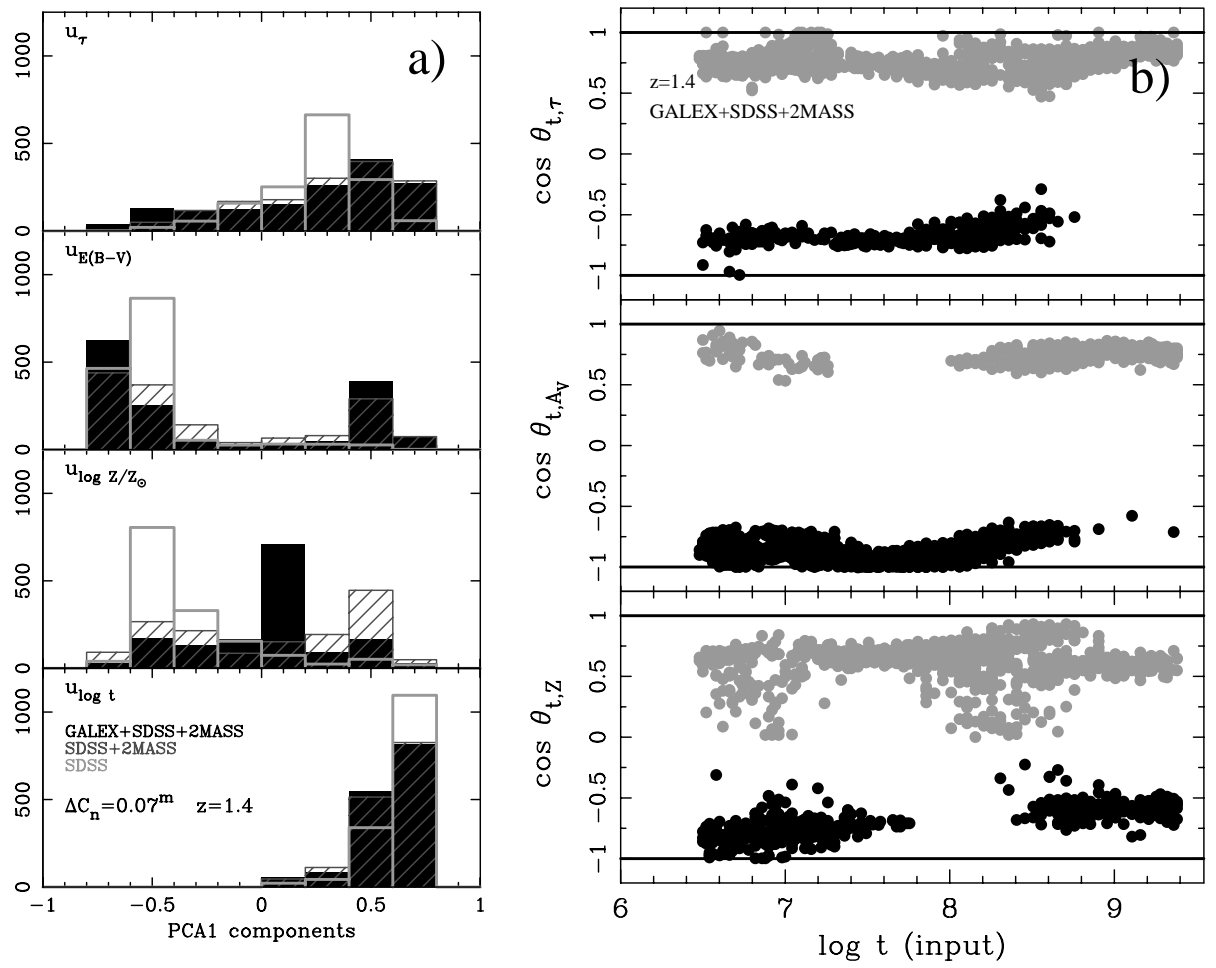

FIG. 4. - (a) Frequency histograms for the components of the PCA1 vectors for the sample of high-redshift galaxies with $\Delta C_{n}=0.07$ mag for three different sets of observables (SDSS, SDSS + 2MASS, and GALEX + SDSS + 2MASS), suggesting that the age-metallicity and age-extinction degeneracies are comparable for the SDSS set, while the age extinction and the age-timescale degeneracies are competing in the case of the SDSS + 2MASS and GALEX + SDSS + 2MASS sets. (b) Cosine of the angle between the PCA1 vector and the age-timescale (top), age-extinction (middle), and age-metallicity (bottom) planes, as a measure of the degeneracy between the stellar population properties (see $\S 4$ ), as a function of the age for high-redshift galaxies with an uncertainty $\Delta C_{n}=0.07 \mathrm{mag}$ and the GALEX + SDSS $+2 \mathrm{MASS}$ set. The sign of the cosine indicates whether the degeneracy is in the sense that an increase in both properties can lead to the same observational properties (positive) or that the value for one of the magnitudes has to be decreased while the other is increased (negative). The dependence of the dominant degeneracy on the age of the stellar population, going from the age-metallicity and age-extinction degeneracies at ages below $10 \mathrm{Myr}$ to only the age-extinction degeneracy at ages between $10 \mathrm{Myr}$ and $300 \mathrm{Myr}$, and the age-timescale degeneracy at ages between 300 Myr and $3 \mathrm{Gyr}($ see $\S 4.3 .2)$ is clear.

observed even for the largest observing errors considered indicates that it is real and a natural consequence of the use of broadband data. In this sense, the combination of broadband with narrow- or medium-band data or spectroscopic indexes would break the age-extinction degeneracy, making age-metallicity the dominant degeneracy (see Worthey 1994).

\subsubsection{Dust Extinction}

The dust extinction is derived with a very high accuracy $[E(B-V)=0.04-0.20 \mathrm{mag}]$ even for large observing errors and a relatively low number of observables (see Table 3 ). In the case of the nearby galaxies, the uncertainty in the dust extinction does not depend on the value of the dust extinction itself and is mainly degenerate with the age of the stellar population (see above), with some contribution from the extinction-metallicity degeneracy. In combination with $B V R I$ optical data the use of either UV, $U$, or NIR data provides a significant reduction in the dust extinction uncertainty. To better derive the dust extinction the use of a wider wavelength baseline in wavelength (e.g., by using $U V I J K$ ) is more effective than fully covering the optical range $(U B V R I)$. This is mainly due to the reduction in the metallicity uncertainty by the use of NIR data (see below) that leads, via the extinction-metallicity degeneracy, to a reduction in the dust extinction uncertainty. Again, the use of
$J H K$ data instead of only $K$-band data does not lead to a significant reduction in the dust extinction uncertainties.

\subsubsection{Metallicity}

With regard to the metallicity of the stellar population the uncertainties derived are strongly dependent on the bandpass combination available and the value of the metallicity itself. In particular, the uncertainties derived are smaller as the metallicity becomes higher (see Fig. 5a). Within the age range considered, the main contributors to the optical and NIR emission of SSP galaxy are the mainsequence and red giant branch (RGB) stars. However, for a more constant star formation, a significant contribution from core-helium-burning stars is expected (see Charlot \& Bruzual 1991). To determine the source of the metallicity dependence of these uncertainties we have produced the same diagrams shown in Figure $5 a$ but restricted to formation timescales shorter than $50 \mathrm{Myr}$. The analysis of this diagram shows no dependence of the uncertainties on metallicity, which implies that the source of the dependence was the distinct photometric evolution of high-metallicity core-helium-burning stars (Mowlavi et al. 1998). It is worth noting, however, that at very high metallicities the uncertainties in the modeling of the stellar populations are themselves very large because of the lack of very metal-rich stars 

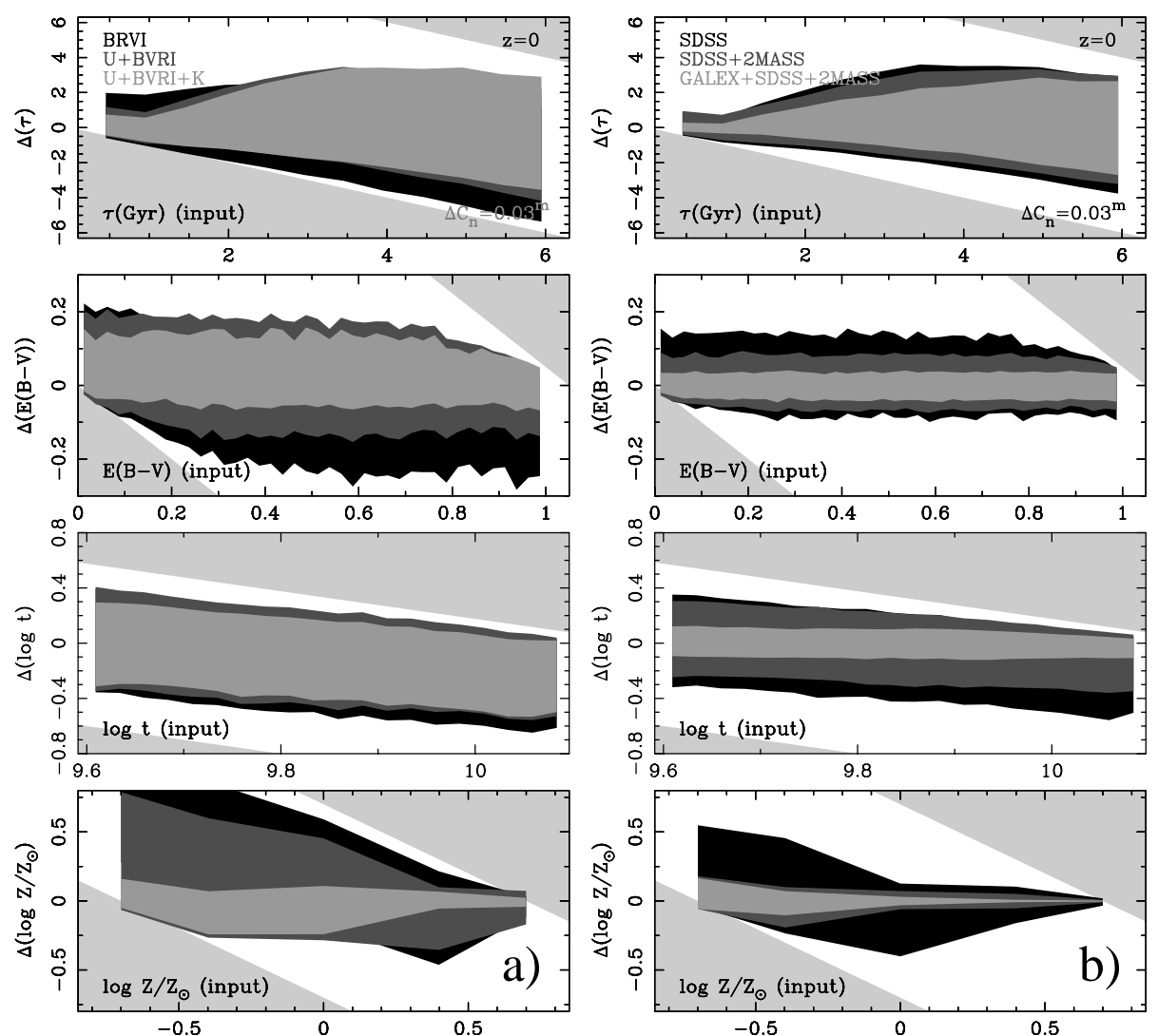

FIG. 5.-Uncertainties in the derived properties of nearby galaxies for (a) the $B V R I, U+B V R I$, and $U+B V R I+K$ sets and $(b)$ the SDSS, SDSS + 2MASS, and GALEX + SDSS + 2MASS sets, assuming observing errors of 0.03 mag.

of any age in the solar neighborhood that could be used as spectral calibrators (see Charlot et al. 1996).

The most significant reduction in the mean metallicity uncertainty is achieved when NIR data are used in combination with optical data (see Fig. 5a). Although the uncertainties in the model predictions for the thermally pulsating AGB and the upper RGB can result in differences in the $V-K$ color predicted by different models of $\sim 0.10-00.15$ mag (Charlot et al. 1996), the improvement in the metallicity determination by the use of NIR data is still relevant. In this sense, in Table 3 we show that the mean metallicity uncertainty for the $U+B V R I$ set is 0.32 dex, assuming an observing error of $0.03 \mathrm{mag}$, while the uncertainty for the $U+B V R I+K$ set, assuming an observing error of 0.10 mag, is only 0.26 dex.

\subsubsection{Stellar Mass}

As input for the $K$-band mass-to-light ratio of the stellar populations we have adopted $M_{K \odot \odot}=3.33$ (Worthey 1994). It should be noted that, along with the errors in the stellar mass-to-light ratios derived here, the misunderstanding of the actual IMF introduces an additional systematic uncertainty that, in fact, constitutes the most important source of error in the determination of the galaxy stellar mass (Bell \& de Jong 2001). In addition, the poor constraints on the theoretical isochrones of upper RGB stars and AGB stars can result in a $20 \%$ uncertainty in the $K$-band mass-to-light ratio (Charlot et al. 1996). In Figure 6 we show the uncertainties expected in the $K$-band mass-to-light ratio from different sets of observables that include $K$-band data. These uncertainties show a strong dependence on the galaxy age and formation timescale in the sense that larger uncertainties are expected at lower values of the formation timescale and older ages. Figure $5 a$ shows that the value of the age uncertainty (in $\log t$ scale) is almost independent of the age itself. In addition, Figure 1 indicates that the rate of change in the $K$-band mass-to-light ratio (with $\log t$ ) is higher when the stellar population becomes older, especially for very low values of the formation timescale. Therefore, for a constant uncertainty in $\log t$, an increase in the uncertainty of the mass-to-light ratio at very old ages is expected.

Figure $6 a$ also shows that the mass-to-light ratio determination is biased toward lower values. This bias, which is particularly important at old ages, is probably due to the upper limit of $15 \mathrm{Gyr}$ in age imposed during the data-model comparison (see Table 1), although other contributors cannot be ruled out (see $\S \S$ 4.2.2 \& 4.3.2). As is clearly seen in Figure 6b, the use of UV data allows us to reduce both the uncertainty and bias in the massto-light determination. This reduction is directly related to the reduction in the age uncertainty described above. However, as we already commented, the use of UV data for the study of stellar populations with ages older than several gigayears can lead to wrong conclusions because of the uncertain modeling of the post-AGB phase and the UV upturn.

The behavior described above for the timescale, age, dust extinction, metallicity, and stellar mass is identical for any observing error but has larger mean uncertainties for larger 

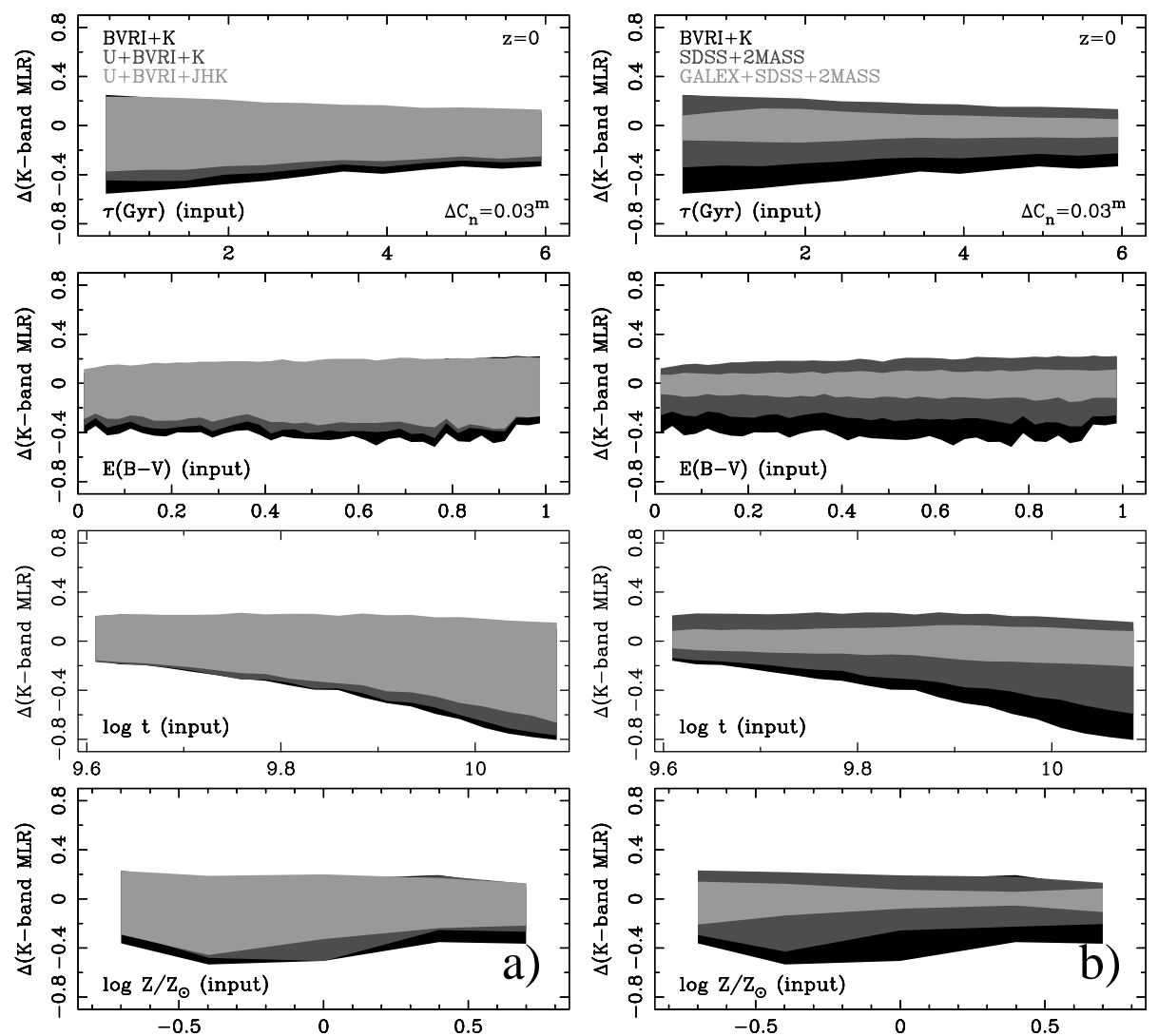

FIG. 6.-Uncertainties in the derived $K$-band mass-to-light ratios for (a) the $B V R I+K, U+B V R I+K$, and $U+B V R I+J H K$ sets and $(b)$ the $B V R I+K$, SDSS + 2MASS, and GALEX + SDSS + 2MASS sets, assuming observing errors of 0.03 mag.

observing errors. The reader is referred to Table 3 for the dependence of the mean uncertainties in the different galaxy properties derived on the observing errors.

\subsection{Intermediate-Redshift Galaxies}

\subsubsection{Formation Timescale}

In Figure 7 we show the uncertainties derived for the properties of intermediate-redshift galaxies $(z=0.7)$. With regard to the formation timescale the uncertainties are very large (2-3 Gyr), even larger than for the nearby galaxy sample. As we commented in $\S 4.1$, the optical-NIR colors are quite insensitive to changes in the formation timescale, with $t \ll \tau$. Therefore, since we are assuming that these galaxies are statistically younger than the those observed in our local universe (see Table 1) and the range in formation timescale is obviously the same, the uncertainty in the formation timescale is necessarily higher. For the same reason, the uncertainty at very low timescale values is much lower than at high timescale values.

Figure $7 a$ (top) also suggests a significant bias in the timescale determination toward lower values of this property. This bias is also the consequence of the small changes in the optical-NIR colors of these galaxies with the timescale when the age is younger than the timescale value. In this case, the higher rate of change in the colors toward lower formation timescales systematically leads to lower values to reproduce the probability distribution associated with the observing errors. It is worth noting that, because of the reduction of this bias, the use of a larger number of bands may result in some cases in a higher timescale uncertainty (see Table 3 for the results for the $U+B V R I+K$ and $U+B V R I+J H K$ sets). As in the nearby galaxies case, the dominant degeneracy involving the formation timescale is the age-timescale degeneracy, in the sense that older galaxies with high formation timescales have colors similar to younger galaxies with a more instantaneous star formation. This is true for any bandpass combination considered. With regard to the optimal set of observables, Table 3 demonstrates that for the same number of bands the use of wider wavelength baselines results in lower uncertainties. In particular, the use of the UVIJK set reduces the timescale, age, and metallicity uncertainties inherent in the $U B V R I$ set, also providing a much lower dust extinction uncertainty than the $B V R I+K$ set. On the other hand, the SDSS + 2MASS and GALEX + SDSS + 2MASS sets result in very similar uncertainties (see Table 3), which implies that the optical and NIR bands provide most of the information available in the UV and in the blue part of the optical spectrum about the galaxy age, star formation history, and metallicity.

\subsubsection{Age}

With respect to the age of the intermediate-redshift galaxies the uncertainties derived are significantly smaller than those in the nearby galaxies case. This is mainly due to the higher rate of change in the rest-frame optical colors within the age range assumed for these galaxies compared with that assumed for the nearby galaxies (see Table 1 and Fig. 1). In addition, the fact that the $K$ band now corresponds to the 

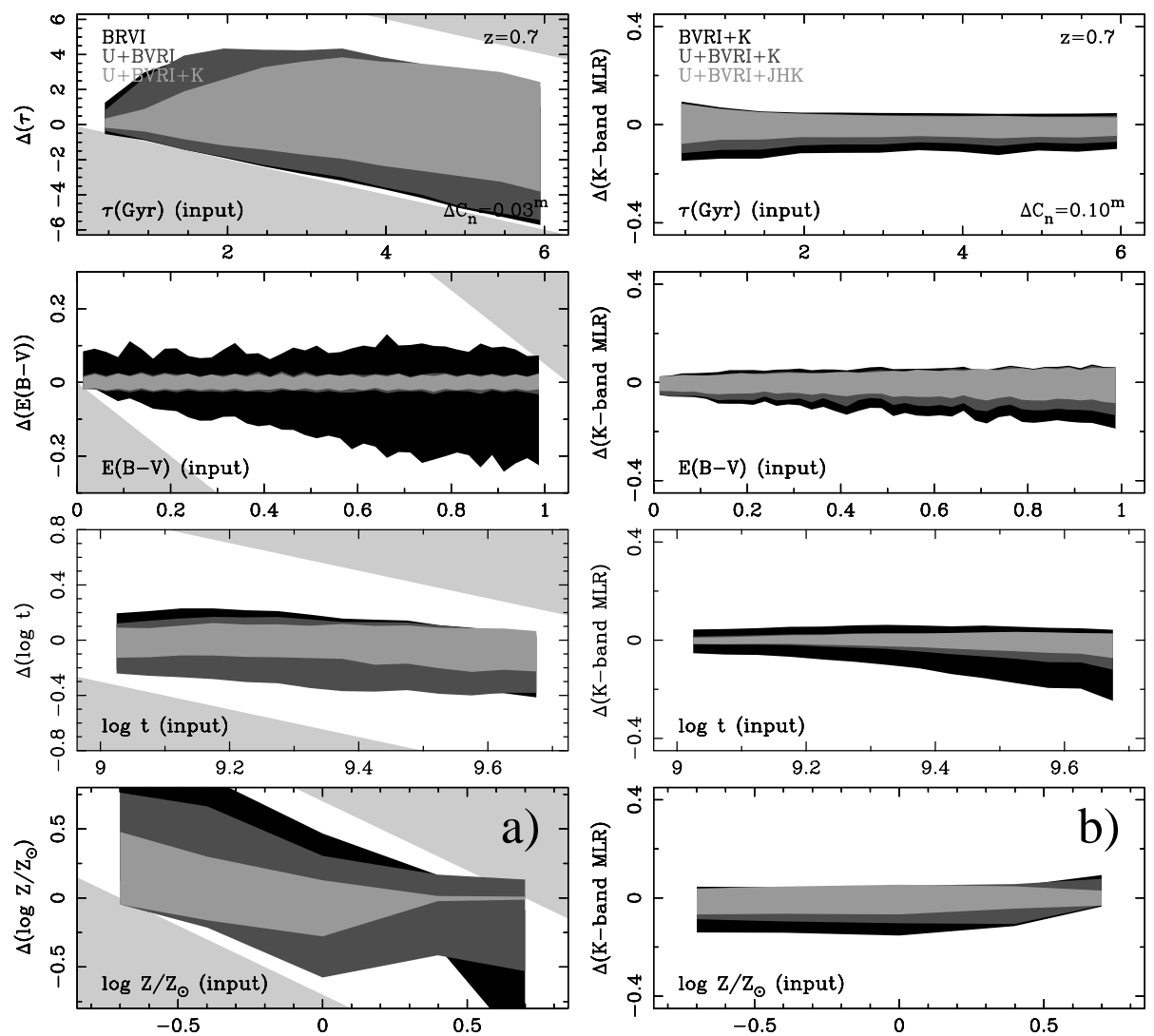

FIG. 7.-(a) Uncertainties in the derived properties of intermediate-redshift galaxies for the $B V R I, U+B V R I$, and $U B V R I+K$ sets, assuming observing errors of $0.03 \mathrm{mag}$; (b) $K$-band mass-to-light ratio uncertainties for the $B V R I+K, U+B V R I+K$, and $U+B V R I+J H K$ sets, assuming observing errors of $0.10 \mathrm{mag}$.

rest-frame $J$-band emission implies that the effect of the uncertainties in the model predictions associated with the upper RGB and AGB evolutionary stages is less important (see $\S$ 4.4.2). On the other hand, the use of $U$-band data for determining ages older than $1 \mathrm{Gyr}$ at these redshifts is strongly limited by the uncertainty in the modeling of the rest-frame UV emission from post-AGB stars (Charlot et al. 1996; see $\S 4.1$ ). However, the most significant decrease in the age uncertainty is achieved when NIR data are used, especially if data in all the bands $(J H K)$ are available. This is probably due to the fact that the $J H K$ set provides information simultaneously about the presence of AGB stars (via the rest-frame $z^{\prime}$ and $J$ bands) and main-sequence stars (via the rest-frame $R$ band).

Figure $5 a$ also shows the existence of a significant bias toward younger ages for the $B V R I$ and $U B V R I$ sets. In this case the presence of this bias is due (1) to the existence of a formation timescale bias and a strong age-timescale degeneracy and (2) to the fact that the optical colors of the stellar populations change more slowly as the population becomes older. In the latter case, to reproduce the distribution of optical colors associated with the observing errors, the bestfitting solution should be found at younger ages, where the intrinsic dispersion of the model colors is larger. As we show below a bias in age also results in a bias in the galaxy $K$-band mass-to-light ratio. The use of wider wavelength baselines allows us to significantly reduce this bias. In particular, the use of the UVIJK leads to a less severe bias and lower age uncertainties than the use of the $U+B V R I$ and
$U+B V R I+K$ sets. Within the age uncertainty interval the degeneracy is mainly dominated by the age-timescale degeneracy, with some contribution from the age-extinction degeneracy in those bandpass combinations that do not include UV- or $U$-band data.

\subsubsection{Dust Extinction}

The dust extinction in the sample of intermediate-redshift galaxies is derived with high accuracy, especially when $U$ band data are available (see Fig. $7 a$ ). In this case, the uncertainties expected in $E(B-V)$ are in any case smaller than $0.10 \mathrm{mag}$ for observing uncertainties as high as $\Delta C_{n}=0.10$ mag. The significant reduction achieved, if we compare these results with those derived for the nearby galaxies, is due to the very high sensitivity of the redshifted UV emission to the presence of small amounts of dust. In bandpass combinations not including $U$-band data we note a clear dependence of the dust extinction uncertainty on the value of the extinction itself, with larger uncertainties at larger values of the extinction (see Fig. 7a). The analysis of the PCA1 components also indicates that at dust extinction values higher than $E(B-V)>0.5$ mag the age-extinction degeneracy becomes very important. This implies that in highly extincted intermediate-redshift galaxies a small increase in the amount of dust can lead to the same optical colors (especially if $U$-band data are not used) as those a comparable decrease in the age of the stellar population would produce. 


\subsubsection{Metallicity}

With regard to the metallicity uncertainty, Figure $7 a$ shows that the uncertainty decreases with the value of the metallicity itself. The reduction is particularly important when NIR data are available. The use of the three $J H K$ NIR bands reduces this uncertainty over the whole range of metallicities. In this sense, the use of the UVIJK set results in lower metallicity uncertainties than the $U+B V R I$ or the $U+B V R I+K$ sets (see Table 3 ). It is important to keep in mind that the $J H K$ filters now cover the rest-frame $R, z^{\prime}$, and $J$ bands. In the age range considered the main contribution to the rest-frame optical emission comes from main-sequence stars. On the other hand, the rest-frame NIR emission, along with that of main-sequence stars, shows an important contribution from AGB and core-helium-burning stars (see Charlot \& Bruzual 1991). The role played by AGB stars is more relevant if the formation is instantaneous, while the corehelium-burning stars may dominate the total NIR emission for a more constant star formation scenario. Therefore, the behavior described above is probably due to the distinct evolution of high-metallicity AGB stars (see Willson 2000 and references therein) and core-heliumburning stars (Mowlavi et al. 1998) compared with the relatively well-defined sequence in their evolutionary properties established for subsolar metallicities. Within the error intervals derived, the metallicity is mainly degenerate with the age, especially in those sets including $U$-band data. This is probably due to the reduction in the age-extinction degeneracy thanks to the information provided by the $U$-band data about the rest-frame UV.

\subsubsection{Stellar Mass}

The comparison between Figures $6 b$ and $7 b$ shows that the mean uncertainties in the $K$-band mass-to-light ratio (or stellar mass) of intermediate-redshift galaxies are much lower than those derived for the nearby sample. First, it is important to note that in these figures we represent absolute errors. For a solar-abundant $12 \mathrm{Gyr}$-old nearby galaxy formed instantaneously the $K$-band mass-to-light ratio is $\sim 1.3 M_{\odot} / L_{K, \odot}$, while for a $5 \mathrm{Gyr}-$ old galaxy at $z=0.7$ it is $\sim 0.4 M_{\odot} / L_{K, \odot}$. Therefore, the relative uncertainties, assuming the average absolute uncertainties given in Table 3 for $\Delta C_{n}=0.07 \mathrm{mag}$, would be about $30 \%$ and $20 \%$, respectively, for the nearby and intermediate-redshift galaxies. Although this still implies a significant improvement in the $K$-band mass-to-light ratio determination, it is also notable that the $K$ filter now traces the rest-frame $J$-band luminosity, which is more affected by the misunderstanding about the actual IMF (see Bell \& de Jong 2001). Finally, the $J$ band luminosity is also more sensitive to small differences between the assumed exponential star formation and the galaxy actual star formation history than the rest-frame $K$-band data.

As we pointed out in $\S 4.2 .2$ the bias in the age determination toward lower age values also leads to a strong bias in the $K$-band mass-to-light ratio of intermediate-redshift galaxies due to the systemic decrease in the rest-frame $J$-band
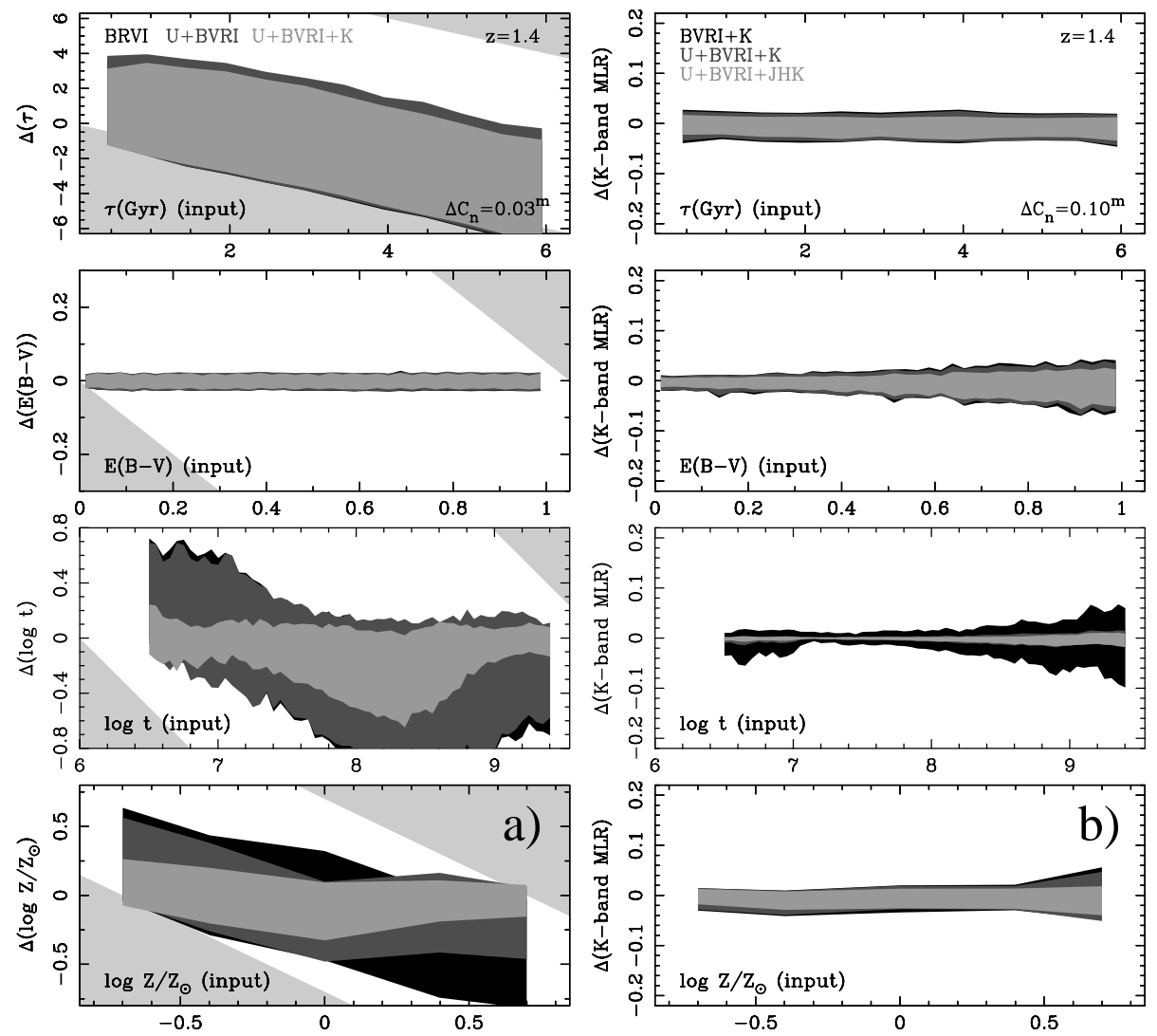

FIG. 8. - (a) Uncertainties in the derived properties of high-redshift galaxies for the $B V R I, U+B V R I$, and $U B V R I+K$ sets, assuming observing errors of $0.03 \mathrm{mag}$; (b) $K$-band mass-to-light ratio uncertainties for the $B V R I+K, U+B V R I+K$, and $U+B V R I+J H K$ sets, assuming observing errors of 0.10 mag. 
luminosity per solar mass with the age of stellar population when the age is older than $\sim 1 \mathrm{Gyr}$.

\subsection{High-Redshift Galaxies}

\subsubsection{Formation Timescale}

With regard to the formation timescale, Figure $8 a$ shows that the bias toward lower timescale values observed at intermediate redshift is even more pronounced at high redshift. This bias is a natural consequence of the difficulty of deriving or predicting the long-term star formation history of a galaxy when it is still very young. This is also evidenced by the fact that the mean timescale uncertainty increases systematically with redshift for the same observing errors and bandpass combinations. In Table 3 we also show that in many cases ( $B V R I$ vs. $U+B V R I$ and SDSS vs. SDSS + 2MASS) the mean timescale uncertainties increase when a larger number of observing bands is used, with a progressive reduction in this bias. As in the intermediateredshift case the dominant degeneracy involving the galaxy formation timescale occurs with the age of the stellar population.

\subsubsection{Age}

The large formation timescale uncertainty described above and the existence of a strong age-timescale degeneracy, especially at ages older than $100 \mathrm{Myr}$, lead to very large age uncertainties, even larger than those derived for the intermediate-redshift galaxies. The age-timescale degeneracy at ages younger than $100 \mathrm{Myr}$ is significantly smaller because at these young ages a change in the formation timescale, which ranges between $200 \mathrm{Myr}$ and 6 Gyr (see Table 1), does not affect the UV-optical-NIR colors of the stellar population. In other words, the degeneracy in timescale within this age range is complete, and no correlation between the age uncertainty and any other uncertainty is expected. In this case the main degeneracies are the ageextinction and the age-metallicity ones.

Moreover, the age-timescale degeneracy, in combination with the bias in formation timescale described above, is also responsible for the strong bias in age observed in Figure $8 a$ at ages older than $\sim 50 \mathrm{Myr}$. The fact that the UVIJK set provides a better age and timescale determination than the $U+B V R I$ and $U+B V R I+K$ sets demonstrates the importance of obtaining $J H K$ data to derive the properties of high-redshift galaxies. This is due to the fact that the $J H K$ filters now cover the rest-frame $V, R$, and $z^{\prime}$ optical bands, where the changes due to galaxy evolution are more noteworthy and the information content about the galaxy properties is greater. In particular, the $J H K$ filters provide information simultaneously about the presence of mainsequence stars (via the rest-frame $V$ and $R$ bands), corehelium-burning stars (via the rest-frame $V, R$, and $z^{\prime}$ bands), and AGB stars (via the rest-frame $z^{\prime}$ band; $t>0.5 \mathrm{Gyr}$ ).

\subsubsection{Dust Extinction}

Because of the extensive coverage of the UV range of the spectrum, the study of high-redshift galaxies by using optical-NIR colors leads to very small dust-extinction uncertainties. In this sense, the dust-extinction uncertainties given in Table 3 at this redshift, assuming an observing error of 0.10 , mag are in the range $E(B-V)=0.03-0.07 \mathrm{mag}$. The dust extinction within the interval of uncertainty is mainly degenerate with the age of the stellar population.

\subsubsection{Metallicity}

Figure 8 shows that the metallicity uncertainty for the high-redshift sample does not show the strong metallicity dependence found in nearby and intermediate-redshift samples. Only when $J H K$ NIR data are available do the uncertainties at very high metallicities become significantly smaller than those derived for the low-metallicity galaxies. As we commented in $\S 4.2 .4$ for the intermediate-redshift case, this is probably due to the distinct signature of highmetallicity core-helium-burning stars (e.g., in the number ratio of blue to red supergiants; Mowlavi et al. 1998) within the age range considered. In the case of an SSP galaxy, these stars dominate the rest-frame $V R z^{\prime}(J H K$ at $z=1.4$ ) emission for ages younger than $0.4 \mathrm{Gyr}$, while the emission at shorter wavelengths comes mainly from main-sequence stars (see Charlot \& Bruzual 1991). It is important to note that the core-helium-burning stars may dominate the emission in the $R$ and $z^{\prime}$ bands up to ages of $5 \mathrm{Gyr}$ for larger formation timescales. Within the uncertainty intervals obtained, the metallicity is mainly degenerate with the age of the stellar population.

\subsubsection{Stellar Mass}

The $K$-band mass-to-light ratio uncertainties derived here are very small compared with those obtained from the nearby and intermediate-redshift samples, with values ranging between 0.01 and $0.06 M_{\odot} / L_{K, \odot}$. If we adopt a $K$-band mass-to-light ratio of $0.27 M_{\odot} / L_{K, \odot}$, which corresponds to the value expected for a $3 \mathrm{Gyr}-$ old galaxy with solar metallicity, the relative uncertainty would range between $5 \%$ and $20 \%$, depending on the bandpass combinations available. Figure $8 b$ shows that there is also a strong dependence of the mass-to-light ratio uncertainty on the value of the massto-light ratio itself. In particular, a clear minimum in its uncertainty is observed at ages older than $8 \mathrm{Myr}$, which is probably associated with the evolution of the massive stars off the main sequence toward the red supergiant phase. During this part of the evolution a sudden change in the rest-frame $z^{\prime}$ luminosity and optical colors of an SSP is produced, which could explain why the uncertainty is particularly small around this age value.

\subsection{Effects of the Model Uncertainties}

In this section we analyze the results obtained when the optical-NIR colors of a sample of galaxies generated by using the GISSEL99 models are compared with the predictions of the PEGASE evolutionary synthesis models. We have restricted this comparison to the nearby sample and the range of properties specified in Table 1. The results of this comparison are shown in Figure 9.

\subsubsection{Formation Timescale}

Figure $9 a$ shows that the same bias toward lower values of the formation timescale that we noted for the intermediate- and high-redshift samples is also present in this case (see $\S \S 4.2 .1$ and 4.3.1). The main reason for the existence of this bias is the small change in the optical-NIR colors of the stellar population with the timescale when the age $t \ll \tau$. Therefore, to compensate for both the observing errors and the differences in the color predictions between the GIS- 

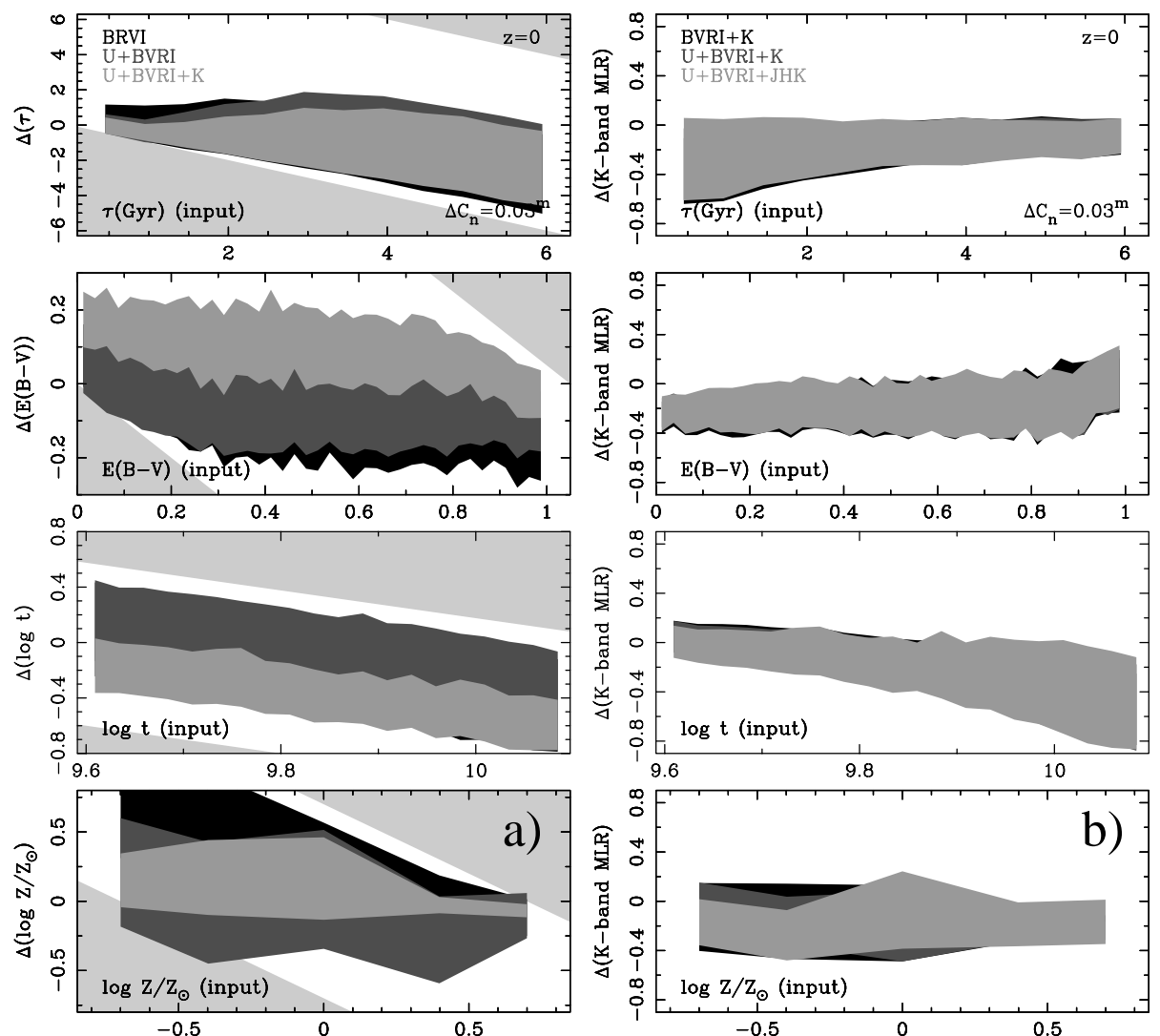

FIG. 9.- (a) Mean timescale, dust extinction, age, metallicity, and $K$-band mass-to-light ratio; $(b)$ uncertainties derived using the PÉGASE evolutionary synthesis models. In (a) we show the results for the $B V R I, U+B V R I$, and $U B V R I+K$ sets, and in $(b)$ those for the $B V R I+K, U+B V R I+K$, and $U+B V R I+J H K$ sets. In both cases observing errors of 0.03 mag have been adopted.

SEL99 and PÉGASE models, the best-fitting solution has to be found at lower values of the timescale for which the intrinsic dispersion of the colors is larger. The existence of this strong bias also leads to very small timescale uncertainties compared with those obtained by using the GISSEL99 models. Within the uncertainty intervals derived, the dominant degeneracy involving the galaxy formation timescale is the age-timescale degeneracy.

\subsubsection{Age}

With regard to the age determination, the uncertainties derived are very similar for the $B V R I$ and $U+B V R I$ sets. However, for bandpass combinations including NIR data the ages derived are strongly biased toward younger ages. The reason for this bias, which also leads to significantly smaller age uncertainties, is the offset in the $J-H$ and $H-K$ colors between the GISSEL99 and the PEGASE model predictions (see Fig. 1), due to the differences in the modeling of the upper RGB and AGB phases. In particular, Figure $1 b$ shows that the PÉGASE models are $\sim 0.07$ mag redder in $J-H$ and $\sim 0.04$ mag redder in $H-K$ than the GISSEL99 models within the age range 4-12 Gyr. Therefore, to compensate for this difference in color, the best-fitting solution usually leads to younger ages, which within this age range imply bluer colors. Because the differences in the colors between the two models occur only in the NIR, the optical colors predicted by the PÉGASE models at these younger ages should be bluer than those of the sample. Therefore, to compensate for this effect, the age bias described above has to be accompanied by strong biases in dust extinction and/ or metallicity that would lead to redder optical colors. Within the error intervals derived the total degeneracy is dominated by the age-timescale and age-extinction degeneracies.

\subsubsection{Dust Extinction}

As we commented above (see also Fig. $9 a$ ) there is a strong bias in dust extinction estimates toward higher extinction values when NIR data are used. This bias, along with the metallicity bias described in $\S 4.4 .4$, results in a global reddening of the optical colors but a small change in the NIR colors of the galaxies in the sample. On the other hand, at very high extinction values the uncertainties are also biased by the upper limit in $E(B-V)$ imposed during the data-model comparison procedure (see Table 1). The mean uncertainties derived in age, dust extinction, and metallicity are very similar to those obtained by using the GISSEL99 models.

\subsubsection{Metallicity}

The distribution of the uncertainty in metallicity shown in Figure $9 a$ indicates that a strong bias toward higher metallicity values is present when NIR data are available. As we commented in $\S 4.4 .2$, this bias is probably related to the age bias and the differences in the NIR colors predicted by the two sets of models. As in the case of GISELL 99 models, the comparison with the PÉGASE models leads to a clear dependence of the uncertainty on the metallicity value itself, 
with smaller uncertainties at very high metallicities (see $\S 4.1 .4)$.

\subsubsection{Stellar Mass}

The results shown in Figure $9 b$ with regard to the $K$-band mass-to-light ratios mainly reflect the biases in the galaxy property determination, with the derived stellar masses systematically smaller than the input values. This is due (1) to the bias toward younger ages described in $\S 4.4 .2$ and (2) to the higher $K$-band luminosity per unit mass of the PÉGASE models compared with the GISSEL99 models (see Fig. 1b). Because of the stronger bias in age, the mean uncertainties in the $K$-band mass-to-light ratios are smaller than those obtained by using the GISSEL99 models (see $\S$ 4.1.5). Finally, Figure $9 b$ shows that the mass-to-light ratio uncertainty becomes higher at older ages and lower timescale values. This behavior, which is also present in the GISSEL99 models (see $\S 4.1 .5$ ), is due to the progressive increase in the rate of change of the $K$-band mass-to-light ratio with $\log t$ (see Fig. 1), accompanied by a small dependence of the age uncertainty (in $\log t$ scale) on the value of the age itself.

\section{CONCLUSIONS}

In this study we have analyzed the dependence of the uncertainties and degeneracies in the galaxy properties on different parameters: (1) the combination of bands available, (2) the observing errors, and (3) the galaxy properties themselves (including redshift).

Here we summarize our main results and point out some directions for the optimization of galaxy evolution studies by using broadband photometry data. We describe separately the nearby, intermediate-redshift, and high-redshift cases.

Nearby galaxies.-To determine the star formation history, age, and dust extinction of nearby galaxies with relatively small uncertainties, the use of $U$-band data is fundamental. The availability of $K$-band data also allows a reduction in the uncertainty in the age and metallicity of the stellar population, but the use of additional $J$ - and $H$-band data is largely redundant. The use of the $K$-band data is unfortunately limited by the existence of large uncertainties in the modeling of the $K$-band luminosities and NIR colors of stellar populations. The most significant reduction in the age and $K$-band mass-to-light ratio uncertainty is achieved when UV data are used. The poor treatment of the postAGB and "extreme" HB phases by the existing evolutionary synthesis models, however, introduces an additional uncertainty during the data-model comparison, which is particularly important in the case of very old stellar populations. For the same number of observing bands, the availability of wider wavelength baselines results in lower uncertainties. Both the formation timescale and $K$-band mass-to-light ratio uncertainties are larger when the corresponding values for these properties are larger. On the other hand, the metallicity uncertainty decreases with the value of the metallicity itself because of the distinct photometric evolution of high-metallicity core-helium-burning stars. A complete description of the physical reasons behind these conclusions and of the degeneracies responsible for the uncertainties described above is given in $\S 4.1$ (see also $\S 4.4)$.
Intermediate-redshift galaxies.-The star formation history of intermediate-redshift galaxies can be derived with poorer precision than in nearby galaxies because their stellar populations are younger. The age uncertainty is smaller than in the nearby galaxies case and shows a strong bias toward younger ages. A significant reduction of this bias and of the mean uncertainties is achieved when NIR data are used, especially if all three of the $J$-, $H$-, and $\mathrm{K}$-band data are available. The dust extinction uncertainty is larger for larger values of the dust extinction itself. The use of $U$-band data provides an important reduction of this dependence and of the mean dust extinction uncertainty. If $U$-band data are available the use of additional UV data does not provide much more information about the galaxy properties. The use of NIR data $(\mathrm{J}-, \mathrm{H}$-, and $\mathrm{K}$-band data) significantly reduces the uncertainty in the metallicity of the galaxy. The absolute and relative uncertainties in the galaxy $K$-band mass-to-light ratio are smaller than those derived for nearby galaxies. However, the fact that the $K$ filter now covers the rest-frame $J$ band leads to a larger uncertainty associated with the IMF and with the parameterization of the galaxy star formation history and, consequently, to a larger stellar mass uncertainty. For a more detailed description see $\S 4.2$.

High-redshift galaxies.-As expected, the bias and mean uncertainty in the determination of the timescale for the galaxy formation are even larger in this case than in the case of the nearby or intermediate-redshift galaxies. The age of the stellar population is derived with a large uncertainty, reduced only when $J H K$ data are available. The dust extinction in these galaxies can be derived to a very high accuracy even when only optical data are available. The use of $J H K$ data is fundamental to improve both the age and metallicity determinations. A complete description of the uncertainties and degeneracies between these properties is given in $\S 4.3$.

Some of the conclusions drawn above can also be found through the literature expressed in a qualitative way. However, this work constitutes the first systematic and quantitative study of the optimization of broadband photometry for studies of the evolution of galaxies. It is important to note that the application of these results to future galaxy surveys can help reduce the uncertainty in the derivation of the galaxy physical properties, sometimes by weakening a particular degeneracy, but most of the time by decreasing the intervals over which this degeneracy takes place. In Table 3 we have summarized the mean uncertainties in the galaxy stellar population properties derived in this paper by considering different redshifts, sets of observables, and observing errors.

Our results are directly applicable to spectrophotometric surveys such as the SDSS and surveys looking for emissionline galaxies at fixed redshifts (Martin, Lotz, \& Ferguson 2000; Moorwood, van der Werf, Cuby \& Oliva 2000; Iwamuro et al. 2000; Pascual et al. 2001; Zamorano et al. 2002). However, in the case of the blind-redshift surveys a comparison between our results and those from previous studies on the optimization of the photometric redshift technique (Kodama et al. 1999; Bolzonella et al. 2000; Mobasher \& Mazzei 2000; Wolf et al. 2001) is still needed.

\section{FUTURE APPLICATIONS}

The results summarized above demonstrate that the design of galaxy evolution studies based only on qualitative, intuitive ideas may lead (in some cases avoidably) to large 
uncertainties. Because of this we intend to apply this work to the design of future projects on galaxy evolution that estimate optimal sets of observables and required signal-tonoise ratios. Although in this work we have considered only broadband filters, this procedure is easily generalizable to combinations of broad-, medium-, and narrowband filters from the far-UV to the near-infrared. In addition, the combined use of the procedure here described with state-of-theart radiative transfer and dust models (Popescu et al. 2000) will allow us to extend this range up to submillimeter wavelengths.

Beyond the results shown in this paper, we can also derive, on request, the uncertainties and degeneracies in the galaxy properties for a given combination of filters, observing errors, and galaxy redshift.

We would like to thank the referee for the useful comments and suggestions that have significantly improved the quality of this paper. We are also very grateful to A. G. Bruzual for providing the GISSEL99 evolutionary synthesis models, P.G. Pérez González, N. Cardiel, and O. Pevunova for stimulating conversations, and C. Sánchez Contreras for carefully reading the manuscript and making some useful remarks. A. G. de P. acknowledges financial support from NASA through a Long Term Space Astrophysics grant to B. F. M.
Abraham, R. G., Ellis, R. S., Fabian, A. C., Tanvir, N. R., \& Glazebrook, K. 1999, MNRAS, 303, 641

Aragón-Salamanca, A., Ellis, R. S., Couch, W. J., \& Carter, D. 1993, MNRAS, 262, 764

Avni, Y. 1976, ApJ, 210, 642

Bell, E. F., \& de Jong, R. S. 2000, MNRAS, 312, 497

.2001, ApJ, 550, 212

Bolzonella, M., Miralles, J.-M. \& Pelló, R. 2000, A\&A, 363, 476

Bressan, A., Fagotto, F., Bertelli, G., \& Chiosi, C. 1993, A\&AS, 100, 647

Brinchmann, J., \& Ellis, R. S. 2000, MNRAS, 536, 77

Brocklehurst, M. 1971, MNRAS, 153, 471

Bruzual, A. G. 2000, in The Evolution of Galaxies. I. Observational Clues, ed. J. M. Vílchez, G. Stasinska, \& E. Pérez (Dordrecht: Kluwer), 221

Calzetti, D. 1997, in AIP Conf. Proc. 408, The Ultraviolet Universe at Low and High Redshift: Probing the Progress of Galaxy Evolution, ed. W. H. Waller (New York: AIP), 403

Calzetti, D., Kinney, A. L., \& Storchi-Bergmann, T. 1996, ApJ, 458, 132

Cardelli, J. A., Clayton G. C., \& Mathis, J. S. 1989, ApJ, 345, 245

Cerviño, M., Gómez-Flechoso, M. A., Castander, F. J., Schaerer, D.

Mollá, M., Knödlseder, J., \& Luridiana, V. 2001, A\&A, 376, 422

Cerviño, M., Luridiana, V., \& Castander, F. J. 2000, A\&A, 360, L5

Charlot, S., \& Bruzual, A. G. 1991, ApJ, 367, 126

Charlot, S., \& Longhetti, M. 2001, MNRAS, 323, 887

Charlot, S., Worthey, G., \& Bressan, A. 1996, ApJ, 457, 625

Connolly, A. J., Szalay, A. S., Dickinson M., Subbarao, M. U., \& Brunner, R. J. 1997, ApJ, 486, L11

Doliber, D. L., Stock, J. M., Jelinsky, S. R., Mally, J., Jelinsky, P. N., Siegmund, O. H., \& Hull, J. S. 2000, Proc. SPIE, 4013, 402

Dove, J. B., Shull, J. M., \& Ferrara, A. 2000, ApJ, 531, 846

Epchtein, N., et al. 1997, Messenger, 87, 27

Ferland, G. J. 1980, PASP, 92, 596

Fioc, M., \& Rocca-Volmerange, B. 1997, A\&A, 326, 950

Fukugita, M., Ichikawa, T., Gunn, J. E., Doi, M., Shimasaku, K., \& Schneider, D. P. 1996, AJ, 111, 1748

Gallego, J., Zamorano, J., Aragón-Salamanca, A., \& Rego, M. 1995, ApJ, $455, \mathrm{~L} 1$

Gallego, J., Zamorano, J., Rego, M., Alonso, O., \& Vitores, A. G. 1996, A\&AS, 120, 323

Gil de Paz, A., Aragón-Salamanca, A., Gallego, J., Alonso-Herrero, A., Zamorano, J., \& Kauffmann, G. 2000, MNRAS, 316, 357 (GIL00)

Iwamuro, F., et al. 2000, PASJ, 52, 73

Karzas, W. J., \& Latter, R. 1961, ApJS, 6, 167

\section{EFERENCES}

Kodama, T., Bell, E. F., \& Bower, R. G. 1999, MNRAS, 302, 152

Leitherer, C., Ferguson, H. C., Heckman, T. M., \& Lowenthal, J. D. 1995 ApJ, 454, L19

Leitherer, C., et al. 1999, ApJS, 123, 3

Madau, P., Dickinson, M. E., \& Pozzeti, L. 1998, ApJ, 498, 106

Madau, P., Ferguson, H. C., Dickinson M. E., Giavalisco M., Steidel C. C., \& Fruchter, A. 1996, MNRAS, 283, 1388

Martin, C., et al. 1997, BAAS, 191(63.04)

Martin, C. L., Lotz, J., \& Ferguson, H. C. 2000, ApJ, 543, 97

Martin, P. G., \& Rouleau, F. 1989, Berkeley EUV Colloq.

Mathis, J. S. 1990, ARA\&A, 28, 37

Mobasher, B., \& Mazzei, P. 2000, A\&A, 363, 517

Moorwood, A. F. M., van der Werf, P. P., Cuby, J. G., \& Oliva, E. 2000, A\&A, 362,9

Morrison, D. F. 1976, Multivariate Statistical Methods (Singapore: McGraw-Hill)

Mowlavi, N., Meynet, G., Maeder, A., Schaerer, D., \& Charbonnel, C. 1998, A\&A, 335, 573

Osterbrook, D. E. 1989, Astrophysics of Gaseous Nebulae and Active Galactic Nuclei (Mill Valley: University Science Books)

Pascual, S., Gallego, J., Aragón-Salamanca, A., \& Zamorano, J. 2001, A\&A, 379, 798

Perez-González, P. G., Gil de Paz, A., Alonso-Herrero, A., Gallego, J., Aragon-Salamanca, A., \& Zamorano, J. 2002, in preparation

Popescu, C. C., Misiriotis, A., Kylafis, N. D., Tuffs, R. J., \& Fischera, J. 2000, A\&A, 362, 138

Ronen, S., Aragón-Salamanca, A., \& Lahav, O. 1999, MNRAS, 303, 284

Skrutskie, M., et al. 1997, in The Impact of Large Scale Near-IR Sky Surveys, ed. F. Garzón, N. Epehtein, A. Omont, B. Burton, \& P. Persi (Dordrecht: Kluwer), 25

Storchi-Bergmann, T., Calzetti, D., \& Kinney, A. L. 1994, ApJ, 429, 572

Willson, L. A. 2000, ARA\&A, 38, 573

Wolf, C., Meisenheimer, K., \& Röser, H.-J. 2001, A\&A, 365, 660

Worthey, G. 1994, ApJS, 95, 107

Yi, S., Demarque, P., \& Oemler, A. 1997, ApJ, 486, 201

York, D. G., et al. 2000, AJ, 120, 1579

Zamorano, J., et al. 2002, in preparation

Zamorano, J., Gallego, J., Rego, M., Vitores, A. G., \& Alonso, O. 1996 ApJS, 105, 343

Zamorano, J., Rego, M., Gallego, J., Vitores, A. G., González-Riestra, R., \& Rodríguez-Caderot, G. 1994, ApJS, 95, 387 